This item was submitted to Loughborough's Research Repository by the author.

Items in Figshare are protected by copyright, with all rights reserved, unless otherwise indicated.

\title{
Gradient dynamics models for liquid films with soluble surfactant
}

PLEASE CITE THE PUBLISHED VERSION

https://doi.org/10.1103/PhysRevFluids.1.083903

\section{PUBLISHER}

(c) American Physical Society.

\section{VERSION}

VoR (Version of Record)

\section{LICENCE}

CC BY-NC-ND 4.0

\section{REPOSITORY RECORD}

Thiele, Uwe, Andrew J. Archer, and L.M. Pismen. 2019. "Gradient Dynamics Models for Liquid Films with Soluble Surfactant”. figshare. https://hdl.handle.net/2134/23456. 


\title{
Gradient dynamics models for liquid films with soluble surfactant
}

\author{
Uwe Thiele* \\ Institut für Theoretische Physik, Westfälische Wilhelms-Universität Münster, Wilhelm Klemm Straße 9, \\ D-48149 Münster, Germany \\ and Center of Nonlinear Science, Westfälische Wilhelms Universität Münster, Corrensstraße 2, \\ 48149 Münster, Germany
}

Andrew J. Archer

Department of Mathematical Sciences, Loughborough University, Loughborough, Leicestershire LE11 $3 T U$, United Kingdom

Len M. Pismen

Department of Chemical Engineering, Technion-Israel Institute of Technology, Haifa 32000, Israel

(Received 6 September 2016; published 23 December 2016)

\begin{abstract}
In this paper we propose equations of motion for the dynamics of liquid films of surfactant suspensions that consist of a general gradient dynamics framework based on an underlying energy functional. This extends the gradient dynamics approach to dissipative nonequilibrium thin-film systems with several variables and casts their dynamic equations into a form that reproduces Onsager's reciprocity relations. We first discuss the general form of gradient dynamics models for an arbitrary number of fields and discuss simple well-known examples with one or two fields. Next we develop the three-field gradient dynamics model for a thin liquid film covered by soluble surfactant and discuss how it automatically results in consistent convective (driven by pressure gradients, Marangoni forces, and Korteweg stresses), diffusive, adsorption or desorption, and evaporation fluxes. We then show that in the dilute limit, the model reduces to the well-known hydrodynamic form that includes Marangoni fluxes due to a linear equation of state. In this case the energy functional incorporates wetting energy, surface energy of the free interface (constant contribution plus an entropic term), and bulk mixing entropy. Subsequently, as an example, we show how various extensions of the energy functional result in consistent dynamical models that account for nonlinear equations of state, concentration-dependent wettability, and surfactant and film bulk decomposition phase transitions. We conclude with a discussion of further possible extensions towards systems with micelles, surfactant adsorption at the solid substrate, and bioactive behavior.
\end{abstract}

DOI: 10.1103/PhysRevFluids.1.083903

\section{INTRODUCTION}

Onsager's evolution equations [1,2], based on the principle of detailed balance embedded in Onsager's reciprocity relations, became a key tool for understanding the relaxational approach to equilibrium in a variety of physical processes. More recently, Doi [3] extended the range of this approach to processes in macroscopic soft matter systems, such as the swelling of gels and the dynamics of liquid crystals. It is less obvious that a similar approach can also be applied to processes out of equilibrium in spatially extended open systems. A well known example is the dynamics of single-layer thin films in the long-wave (or lubrication) approximation [4,5] where a single variable, the layer thickness, is sufficient for a description of the system. In this case, it is not a priori obvious that an energy functional of thermodynamic origin exists for the system. Nevertheless, as noticed

\footnotetext{
*u.thiele@uni-muenster.de; http://www.uwethiele.de
} 
by Mitlin [6] for dewetting films and by Rosenau and Oron for thin films heated from below [7], the dynamic equation for the layer thickness $h$ can be cast into a gradient dynamics form

$$
\partial_{t} h=\nabla \cdot\left[Q^{\mathrm{c}} \nabla \frac{\delta \mathcal{F}}{\delta h}\right]-Q^{\mathrm{nc}}\left(\frac{\delta \mathcal{F}}{\delta h}-p_{\text {vap }}\right),
$$

showing that the evolution can be derived from a certain energy functional $\mathcal{F}[h]$. Here $p_{\text {vap }}$ is the pressure of the vapor phase that may instead be incorporated into $\mathcal{F}$. Here and in the following $\partial_{t}$ denotes a partial derivative with respect to time and $\nabla$ is the two-dimensional (2D) spatial gradient operator. Equation (1) is the general form in which the dynamics has both conserved and nonconserved contributions with mobilities $Q^{\mathrm{c}}(h) \geqslant 0$ and $Q^{\mathrm{nc}}(h) \geqslant 0$, respectively [8].

The usual procedure of irreversible thermodynamics is thereby reversed: First comes a dynamic equation obtained through a series of simplifications and then a suitable functional is assigned, ensuring a dissipative evolution toward a minimum of this energy. However, in the case of dewetting the energy functional is the interface Hamiltonian that is obtained via a systematic coarse-graining procedure from the microscale interaction energies [9]. Sometimes, even systems that are permanently out of equilibrium can be accommodated, as in the case of sliding droplets on an infinitely extended incline, where the correct thin-film model can be brought into the form of a gradient dynamics with an underlying energy functional that includes potential energy [10].

Besides long-wave thin-film equations, other examples of one-field gradient dynamics are the Cahn-Hilliard equation describing the demixing of a binary mixture, i.e., a purely conserved dynamics $\left(Q^{\text {nc }}=0\right)[11-13]$ and the Allen-Cahn equation that models, for instance, the purely nonconserved dynamics $\left(Q^{\mathrm{c}}=0\right)$ of the Ising model in the mean-field continuum limit [13]. In general, equations of the form (1) are ubiquitous. They appear with various choices of $\mathcal{F}$, not only in the context of the dynamics of films of nonvolatile and volatile liquids on solid substrates $[4,6,14,15]$, but also as evolution equations for surface profiles in epitaxial growth [8,16-19] and as models of one-component lipid bilayer adhesion dynamics [20]. Another field of application is in dynamical density functional theory (DDFT), describing the dynamics of the density distribution of colloidal particles [21-24].

Furthermore, many hydrodynamic two- and more-field long-wave models were developed that describe, e.g., the evolution of multilayer films, films of mixtures, or surfactant-covered films [5]. Normally, they are not written in a gradient dynamics form. However, recently, the gradient dynamics approach was extended to several two-field models, namely, for the dewetting of two-layer films [25,26], for the coupled decomposition and dewetting of a film of a binary mixture [27,28], and for the evolution of a layer of insoluble surfactant on a thin liquid film [29]. In all these cases, energies with a clear physical meaning can be given that may also be obtained via the coarse-graining procedures of statistical physics. Note, though, that the description of a thin two-layer film heated from below cannot be brought into the Onsager form [30], marking the single-layer case as a fortuitous accident. Nonetheless, certain out-of-equilibrium phenomena can be described via the addition of appropriate potential energies to the energy functional or, as in the case of dip coating and Langmuir-Blodgett transfer, through comoving frame terms that account for a moving substrate that is withdrawn from a bath [31]. Similar two-field gradient dynamics models exist for the dynamics of membranes [32,33] or as DDFTs for mixtures [34,35].

The aim of this paper is to extend the gradient dynamics approach to describe the nonequilibrium dissipative dynamics of thin-film systems with several variables and to cast the dynamic equations into a form that reproduces Onsager's reciprocity relations. A further aim is to incorporate interphase exchange processes, such as evaporation and surfactant dissolution to derive equations combining conserved (Cahn-Hilliard-type) and nonconserved (reaction-diffusion- or Allen-Cahn-type) terms. The particular example that is treated is a thin liquid film that is covered by a soluble surfactant and rests on a solid substrate, as sketched in Fig. 1. The resulting three-field gradient dynamics model describes the coupled evolution of the film height profile, the amount of surfactant within 


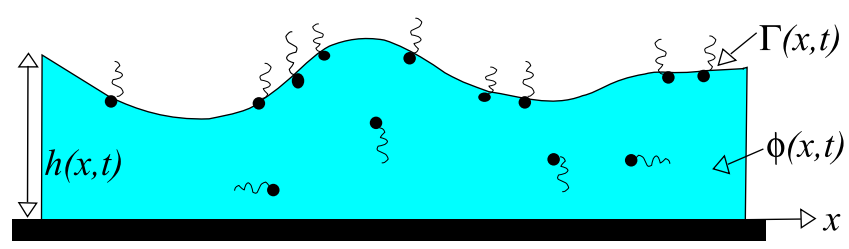

FIG. 1. Sketch of the system under consideration. It consists of a solid planar surface upon which is a film of liquid of thickness $h(x, t)$ that varies with location on the surface $x$ and with time $t$. On the free surface of the liquid film are surfactant molecules, with local density $\Gamma(x, t)$. The surfactant molecules have some solubility in the liquid and the local concentration within the body of the liquid is $\phi(x, t)$. We assume that $\phi$ does not vary vertically and only varies horizontally and with time $t$. This is equivalent to treating $\phi$ as a height-averaged concentration. Over time there is exchange of surfactant molecules between the surface of the liquid and the bulk. There can also be condensation or evaporation of the liquid to vapor in the air above.

the film, and the surface concentration for the case of a thin liquid film covered by a soluble surfactant. The general three-field model recovers several known two-field models as limiting cases, alleviates a number of their shortcomings, and opens up several avenues for systematic extensions.

This paper is structured as follows. In Sec. II A we provide the general form of gradient dynamics models for an arbitrary number of scalar fields with combined conserved and nonconserved dynamics. In Sec. II B we briefly review how to formulate the diffusion equation and the thin-film equation as gradient dynamics and discuss known two-field models. In Sec. III we develop the general three-field gradient dynamics model for a thin liquid film covered by a soluble surfactant. In Sec. IV we discuss special cases and various extensions. We summarize and draw our conclusions in Sec. V. Appendixes A and B clarify an issue in the comparison of the hydrodynamic long-wave approach and the present variational approach and give the variations of the energy functional in the most general case covered by the present work, respectively.

\section{GENERAL $N$-FIELD MODEL AND KNOWN APPLICATIONS}

\section{A. General model}

The dynamics of a spatially extended system may be characterized by the coupled evolution of $N$ scalar state variable fields (order parameter fields) $\mathbf{u}=\left(u_{1}, u_{2}, \ldots, u_{n}\right)^{\mathrm{T}}$. Not too far from equilibrium, the dynamics is governed by a single equilibrium free-energy functional $\mathcal{F}[\mathbf{u}]$, i.e., it is a gradient dynamics. Using Einstein's index notation that presumes summation over repeated indices, the coupled evolution equations read

$$
\partial_{t} u_{a}=\nabla_{\alpha}\left[Q_{a b}^{\mathrm{c}} \nabla_{\alpha} \frac{\delta \mathcal{F}}{\delta u_{b}}\right]-Q_{a b}^{\mathrm{nc}} \frac{\delta \mathcal{F}}{\delta u_{b}},
$$

where $\alpha=1,2, \ldots, d$ refers to spatial coordinates and $a, b=1, \ldots, n$ refer to the different order parameter fields that might have a conserved, nonconserved, or mixed dynamics. Here $Q_{a b}^{\mathrm{c}}(\mathbf{u})$ and $Q_{a b}^{\mathrm{nc}}(\mathbf{u})$ represent $(n \times n)$-dimensional positive-definite and symmetric mobility matrices for the conserved and nonconserved parts of the dynamics, respectively. The mobilities $Q_{a b}^{\mathrm{c}}$ govern the fluxes $j_{a}=-Q_{a b}^{\mathrm{c}} \nabla\left(\delta \mathcal{F} / \delta u_{b}\right)$ of the conserved part of the dynamics for all order parameters $u_{a}$. These are given as linear combinations of the influences of all thermodynamic forces $-\nabla\left(\delta \mathcal{F} / \delta u_{b}\right)$, i.e., are linear in the thermodynamic forces. In contrast, the coefficients $Q_{a b}^{\text {nc }}$ give the transition rates between fields and the resulting nonconserved fluxes are also linear combinations of the thermodynamic potentials $\delta \mathcal{F} / \delta u_{a}$. 
It is straightforward to show that the free energy $\mathcal{F}\left[u_{1}, \ldots, u_{n}\right]$ is a Lyapunov functional, i.e., it monotonically decreases in time:

$$
\begin{aligned}
\frac{d}{d t} \mathcal{F}\left[u_{1}, \ldots, u_{n}\right] & =\int_{\Omega} \frac{\delta \mathcal{F}}{\delta u_{a}} \frac{\partial u_{a}}{\partial t} d^{d} x \\
& =\int_{\Omega} \frac{\delta \mathcal{F}}{\delta u_{a}} \nabla_{\alpha}\left[Q_{a b}^{\mathrm{c}} \nabla_{\alpha} \frac{\delta \mathcal{F}}{\delta u_{b}}\right] d^{d} x-\int_{\Omega} \frac{\delta \mathcal{F}}{\delta u_{a}} Q_{a b}^{\mathrm{nc}} \frac{\delta \mathcal{F}}{\delta u_{b}} d^{d} x \\
& =-\int_{\Omega}\left(\nabla_{\alpha} \frac{\delta \mathcal{F}}{\delta u_{a}}\right) Q_{a b}^{\mathrm{c}}\left(\nabla_{\alpha} \frac{\delta \mathcal{F}}{\delta u_{b}}\right) d^{d} x-\int_{\Omega} \frac{\delta \mathcal{F}}{\delta u_{a}} Q_{a b}^{\mathrm{nc}} \frac{\delta \mathcal{F}}{\delta u_{b}} d^{d} x \leqslant 0,
\end{aligned}
$$

where $\Omega$ is the domain in which the system is defined. Above we used Eq. (2) and partial integration, assuming periodic or no-flux boundary conditions.

A further advantage of the general formulation is the ease with which one may change the choice of variables $u_{a}$. If new order parameter fields $\tilde{u}_{a}$ are introduced via a linear transformation $\tilde{u}_{a}=R_{a b} u_{b}$ the kinetic equations for the new fields are

$$
\partial_{t} \tilde{u}_{a}=\nabla_{\alpha}\left[\widetilde{Q}_{a b}^{\mathrm{c}}\left(\tilde{u}_{1}, \ldots, \tilde{u}_{n}\right) \nabla_{\alpha} \frac{\delta \mathcal{F}}{\delta \tilde{u}_{b}}\right]-\widetilde{Q}_{a b}^{\mathrm{nc}}\left(\tilde{u}_{1}, \ldots, \tilde{u}_{n}\right) \frac{\delta \mathcal{F}}{\delta \tilde{u}_{b}},
$$

with $\widetilde{Q}_{a b}^{\mathrm{i}}=R_{a d} Q_{d e}^{\mathrm{i}} R_{b e}\left(\mathrm{i}=\mathrm{c}, \mathrm{nc}\right.$ ), where we take into account that $\delta \mathcal{F} / \delta u_{a}=R_{b a} \delta \mathcal{F} / \delta \tilde{u}_{b}$. For two conserved fields, similar relations were already given in Refs. [31,36].

Up to here, we have not specified the free energy $\mathcal{F}\left[u_{1}, \ldots, u_{n}\right]$ that can, in principle, be an arbitrary functional of the order parameter fields that is normally bounded from below. If $\mathcal{F}$ is a multiple integral, Eq. (2) becomes a system of integro-differential equations, as is often the case in DDFT for colloids [23]. However, often the kernel is expanded in derivatives of the order parameter fields and Eq. (2) corresponds to a system of partial differential equations. Examples are phase field crystal models [37] and membrane models [33,38] where the highest-order terms in the energy are $\sim(\Delta u)^{2}$. Here we restrict our attention to a lower order and only consider models where the highest-order terms are $\sim(\nabla u)^{2}$. Then the general form is

$$
\mathcal{F}\left[u_{1}, \ldots, u_{n}\right]=\int_{\Omega}\left[\frac{1}{2}\left(\nabla_{\alpha} u_{a}\right) \Sigma_{a b}\left(\nabla_{\alpha} u_{b}\right)+f\left(u_{1}, \ldots, u_{n}\right)\right] d^{d} x,
$$

where we have introduced in the free energy a symmetric, positive-definite $(n \times n)$-dimensional gradient interaction matrix $\Sigma_{a b}$ that, in principle, may itself also depend on $\mathbf{u}$. The integrand may also contain metric factors (see below).

Before we discuss in Sec. III the case of liquid films that are covered with a soluble surfactant, we briefly review in Sec. II B some basic examples where only one or two order parameter fields are involved.

\section{B. Specific known examples of gradient dynamics}

\section{Diffusion equation}

In the dilute limit, the diffusion of a species with part-per-volume concentration $c$ in a quiescent carrier medium can be represented as the conserved gradient dynamics

$$
\partial_{t} c=\nabla \cdot\left[Q_{c c}^{\mathrm{c}} \nabla \frac{\delta \mathcal{F}}{\delta c}\right]
$$

with the purely entropic Helmholtz free-energy functional

$$
\mathcal{F}[c]=\frac{k T}{l^{3}} \int c[\ln c-1] d V,
$$

where $k$ is Boltzmann's constant, $T$ is the temperature, and $l$ is a molecular length scale. The mobility function in Eq. (6) is $Q_{c c}^{\mathrm{c}}=\widetilde{D} c$ and can be obtained via Onsager's variational principle [3,36,39]. 
Here $\widetilde{D}$ is the molecular mobility. This corresponds to $\partial_{t} c=-\nabla \cdot \mathbf{j}_{\text {diff }}$ where Fick's law takes the form $\mathbf{j}_{\text {diff }}=-\widetilde{D} c \nabla \mu=-D \nabla c$ with the chemical potential $\mu=\delta \mathcal{F} / \delta c=\left(k T / l^{3}\right) \ln c$, i.e., $D=\widetilde{D} c d \mu / d c=\widetilde{D} k T / l^{3}$.

The equivalence of Eq. (6) and the standard diffusion equation has been easily shown and now allows one to use the advantages of the gradient dynamics form, namely, the straightforward way to account for free energies that are not purely entropic. If, for instance, one replaces the integrand in $\mathcal{F}[c]$ of Eq. (7) by the sum of a double-well potential and a squared gradient term, one obtains the Cahn-Hilliard equation (then approximating $Q_{c c}^{\mathrm{c}}$ by a constant) [12].

\section{Thin films of simple liquids}

As discussed above, Eq. (1) describes the evolution of the height profile of a thin liquid film on a solid substrate for nonvolatile $\left[Q^{\text {nc }}(h)=0\right.$ ] or volatile $\left[Q^{\text {nc }}(h)>0\right]$ liquids. Detailed discussions of the various physical situations treated can be found in Refs. [4,8,15]. In the most basic case of mesoscopic hydrodynamics, only the influence of capillarity and wettability is considered. The corresponding free energy $\mathcal{F}[h]$ is then

$$
\mathcal{F}[h]=\int\left[\frac{\gamma}{2}(\nabla h)^{2}+g(h)\right] d^{2} x,
$$

where $\gamma$ is the surface tension of the liquid and $g(h)$ is a local free energy (wetting or adhesion energy, or binding potential), related to the Derjaguin (or disjoining) pressure $\Pi(h)$ by $\Pi=-d g(h) / d h[40]$. Note that varying sign conventions are used throughout the literature. ${ }^{1}$ Without slip at the substrate, $Q^{\mathrm{c}} \equiv Q_{h h}^{\mathrm{c}}=h^{3} / 3 \eta$, where $\eta$ is the dynamic viscosity. Different slip models can be accommodated by alternative choices of $Q_{h h}^{\mathrm{c}}$ [45]. Although several functions $Q^{\mathrm{nc}}(h)$ are discussed in the literature for the case of volatile liquids (see, e.g., [15]), often a constant is used [46].

\section{Two-field models}

In the context of thin-film hydrodynamics, two-field gradient dynamics models were presented and analyzed (i) for dewetting two-layer films on solid substrates, i.e., staggered layers of two immiscible fluids [25,26,30], (ii) for decomposing and dewetting films of a binary liquid mixture (with components that are not surface active) [27,28], and (iii) for the dynamics of a liquid film that is covered by an insoluble surfactant [29]. In all three cases, the model has the form (2) with $a, b=1,2$ and all $Q_{a b}^{\mathrm{nc}}=0$ (purely conserved dynamics). The conserved fields $u_{1}$ and $u_{2}$ represent in case (i) the lower layer thickness $h_{1}$ and overall thickness $h_{2}$, respectively [25,26,30], or the lower and upper layer thicknesses [47] [the transformation between the two formulations follows from the discussion around Eq. (4)]. In case (ii), $u_{1}$ and $u_{2}$ represent the film height $h$ and the effective solute height $\psi=c h$, respectively, where $c$ is the height averaged concentration. Finally, in case (iii), $u_{1}$ and $u_{2}$ represent the film height $h$ and the surfactant coverage $\widetilde{\Gamma}$ (which is projected on the Cartesian substrate plane), respectively [29].

A crucial point in cases (ii) and (iii) is to choose two fields that can be varied independently of each other. This is not the case if, e.g., film height $h$ and height-averaged concentration $c$ are used in case (ii), since then a variation in the height for fixed particle number per substrate area implies that $c$ varies [27]. In case (iii), the projected coverage $\widetilde{\Gamma}$ has to be used since the surfactant coverage $\Gamma$ on the free surface and the height profile $h$ are not independent [29]: If the slope of $h$ changes locally, the surface area changes and so does $\Gamma$. Therefore, for a fixed local number of surfactant

\footnotetext{
${ }^{1}$ For particular forms of $\Pi$, see, e.g., Refs. [4,6,14,40-42]. Similar expressions are obtained as interface Hamiltonians in the context of wetting transitions [9]. Therefore, mesoscopic thin-film (or two-dimensional) hydrodynamics might be considered as a gradient dynamics on the underlying interface Hamiltonian. Note that, recently, such mesoscopic wetting energies have been extracted via parameter passing methods from different microscopic models (molecular dynamics and density functional theory) $[43,44]$.
} 
molecules, the local concentration changes without any surfactant transport. If one uses dependent fields, one is not able to employ the general form (2). ${ }^{2}$ In all three cases (i)-(iii) the underlying free-energy functionals have a clear thermodynamic significance. They may be seen as extensions of the interface Hamiltonian for a single adsorbed layer and the individual terms may be obtained from equilibrium statistical physics. As expected, the mobility matrices $\mathbf{Q}^{c}$ are positive definite and symmetric $[25,28,29,47]$. All their entries are low-order polynomials in the respective fields $u_{1}$ and $u_{2}$. In particular, in cases (ii) and (iii), one has

$$
\mathbf{Q}^{\mathrm{c}}=\frac{1}{3 \eta}\left(\begin{array}{cc}
u_{1}^{3} & q u_{1}^{2} u_{2} \\
q u_{1}^{2} u_{2} & r u_{1} u_{2}^{2}+3 \widetilde{D} \eta u_{2}
\end{array}\right),
$$

where $\widetilde{D}$ is a respective molecular mobility related to diffusion, $q=r=1$ in case (ii), and $q=3 / 2$ and $r=3$ in case (iii). ${ }^{3}$

Note in particular that cases (ii) and (iii) in the low-concentration limit give the known hydrodynamic thin-film equations coupled to the equation for the solute or surfactant as discussed in detail in Refs. [27,29], respectively. It recovers also a number of other special cases and can be employed to devise models that incorporate various energetic cross couplings in a thermodynamically consistent manner. Examples include wetting energies that depend on solute or surfactant concentration, effects of surface rigidity for surfactant covered films, and free energies of mixing or demixing including gradient contributions. It also allows one to discuss the influence of solutes or surfactants on evaporation.

Note that the discussion above mixes the possible extensions in cases (ii) and (iii), which are separately discussed in Refs. [27,29], respectively. It was noted in Ref. [28] that the two-field model for a film of a mixture cannot accommodate a solutal Marangoni effect by simply incorporating a concentration-dependent surface tension since this breaks the gradient dynamics structure. Another disadvantage of the two-field model is that most surfactants are soluble, a situation that cannot be treated via case (iii). In the following, we develop a three-field model that alleviates all the mentioned problems.

\section{SOLUBLE SURFACTANT-GRADIENT DYNAMICS MODEL}

\section{A. Energy functional}

We consider a thin film of liquid of thickness $h$ on a solid substrate with a free surface that is covered by a soluble surfactant, i.e., part of the surfactant is dissolved in the bulk of the film and part is adsorbed at the free surface (see Fig. 1). We neglect adsorption at the solid-liquid interface and micelle formation but discuss in the Conclusion how they can be incorporated. The surfactant concentration $\phi$ within the film represents a height-averaged concentration, i.e., it is assumed that the concentration is nearly uniform over the film layer thickness. The system is considered in relaxational situations, i.e., the boundary conditions do not sustain energy or mass fluxes. Therefore, we expect the system dynamics to follow a pathway that approaches a static equilibrium. In the absence of evaporation and surfactant exchange between the interface and the bulk solution, the approach to equilibrium can be described by gradient dynamics for three independent fields: the film thickness $h(\mathbf{r}, t)$, the local amount of dissolved surfactant $\psi(\mathbf{r}, t)=h(\mathbf{r}, t) \phi(\mathbf{r}, t)$, and the surfactant concentration at the interface projected onto a Cartesian reference plane $\widetilde{\Gamma}(\mathbf{r}, t)$. The surfactant concentration on the interface is given by $\Gamma=\widetilde{\Gamma} / \sqrt{a}$, where $a$ is the determinant of the surface metric tensor (see below). Here $\mathbf{r}=(x, y)$ are horizontal coordinates in the substrate plane. The

\footnotetext{
${ }^{2}$ Note that in Refs. [48,49] case (ii) has been treated by employing a gradient dynamics for $h$ and $c$. For a further comparison with the approach employed in Refs. [27,28], see Ref. [15].

${ }^{3}$ Actually, in the parametrization of Ref. [26], the mobility matrix $\mathbf{Q}^{\mathrm{c}}$ of case (i) also agrees with case (iii) if the diffusion term $3 \widetilde{D} \eta u_{2}$ is replaced by $\eta_{\mathrm{r}} u_{2}^{3}$, where $\eta_{\mathrm{r}}$ is the viscosity ratio of the two layers.
} 
fields $\phi$ and $\Gamma$ are expressed as volume fraction and area fraction concentrations, respectively, i.e., they are both dimensionless. As emphasized in Sec. II B 3 for the two-field cases, variations in $h, \phi$, and $\Gamma$ are not independent, while variations with respect to $h, \psi$, and $\widetilde{\Gamma}$ are independent. ${ }^{4}$

The general expression for the energy includes surface and bulk contributions

$$
\begin{gathered}
\mathcal{F}=\mathcal{F}_{\mathrm{s}}+\mathcal{F}_{\mathrm{b}}=\int\left(\mathcal{L}_{\mathrm{s}}+\mathcal{L}_{\mathrm{b}}\right) d x d y, \\
\mathcal{L}_{\mathrm{s}}=\left[\frac{\kappa_{\mathrm{s}}}{2} a^{\alpha \beta}\left(\partial_{\alpha} \Gamma\right)\left(\partial_{\beta} \Gamma\right)+f_{\mathrm{s}}(\Gamma)\right] \sqrt{a}+g(h), \\
\mathcal{L}_{\mathrm{b}}=h\left[\frac{\kappa}{2}|\nabla \phi|^{2}+f(\phi)\right] .
\end{gathered}
$$

The interfacial terms in Eq. (11) depend on the surface metric tensor $a_{\alpha \beta}=\delta_{\alpha \beta}+\partial_{\alpha} h \partial_{\beta} h$ [where we exclude overhangs in order to use a Monge representation for $h(x, y)]$ and its inverse $a^{\alpha \beta} ; a$ is the determinant of $a_{\alpha \beta}$ and determines the extension of the interface and $\delta_{\alpha \beta}$ is the Cartesian metric of the planar substrate or a planar surface $h=$ const. Distinction between lower (covariant) and upper (contravariant) indices is essential for a non-Euclidean surface metric. The wetting potential $g(h)$ in Eq. (11) describes the interactions with the substrate that determine the Derjaguin (or disjoining) pressure $\Pi(h)=-d g(h) / d h$ (cf. Sec. II B 2). The first terms in square brackets in Eqs. (11) and (12) contain the interfacial and bulk rigidity coefficients $\kappa_{\mathrm{S}}$ and $\kappa$, respectively, and penalize surfactant concentration gradients. The second terms in the square brackets in each case take account of molecular interactions. Here $f_{\mathrm{s}}(\Gamma)$ contains the free-energy contribution due to the presence of surfactant molecules at the interface. In the limit $\Gamma \rightarrow 0$, this is just the pure liquid-vapor surface tension, i.e., $f_{\mathrm{s}}(\Gamma \rightarrow 0)=\gamma_{0}$, but more generally

$$
f_{\mathrm{s}}(\Gamma)=\gamma_{0}+\frac{k T}{l_{s}^{2}} \Gamma[\ln \Gamma-1]+f_{s}^{e x}(\Gamma) .
$$

The second term is the contribution to the free energy when the amount of surfactant on the surface is low enough that interactions between molecules are negligible and can be treated as a 2D ideal gas. Here $l_{s}$ is a molecular length scale related to the size of the adsorbed surfactant molecules $\left(l_{s}^{2}\right.$ is the area on the surface occupied by a surfactant molecule). As the surface coverage $\Gamma$ increases, then the excess free energy $f_{\mathrm{s}}^{e x}(\Gamma)$ gives an increasing contribution. For example, treating the surfactant on the surface via a lattice-gas approximation, one would write

$$
f_{\mathrm{s}}^{e x}(\Gamma)=\frac{k T}{l_{s}^{2}}[\Gamma+(1-\Gamma) \ln (1-\Gamma)]-\frac{b}{2} \Gamma^{2},
$$

where the first (entropic) excluded-volume term comes from assuming that only one surfactant molecule can occupy a site of area $l_{s}^{2}$ on the surface and the final term is a simple mean-field term coming from the attraction between pairs of neighboring surfactant molecules. If the attraction strength parameter $b>0$ is sufficiently large, then surface phase transitions may occur. An alternative approximation might be $f_{\mathrm{s}}^{e x}(\Gamma)=f_{h d}(\Gamma)-b \Gamma^{2} / 2$, where $f_{h d}$ is the hard-disk excess free energy (see, for example, the approximations in Refs. [50,51]).

Similarly, the bulk free energy in Eq. (12) can be written as

$$
f(\phi)=\frac{k T}{l^{3}} \phi[\ln \phi-1]+f_{b}^{e x}(\phi),
$$

where $l$ is a molecular length scale related to the surfactant molecules in solution $\left(l^{3}\right.$ is the volume occupied by a surfactant molecule). The simplest approximation is to assume $l_{s}=l$. Here $f_{b}^{e x}(\phi)$

\footnotetext{
${ }^{4}$ Note that the transformation from the dependent fields $h, \phi$, and $\Gamma$ to the independent ones $h, \psi$, and $\widetilde{\Gamma}$ is nonlinear and does therefore not contradict Sec. II A, where we have discussed the possibility of using linear transformations to obtain other sets of independent variables while conserving the gradient dynamics structure.
} 
is the bulk excess contribution, which in general may be written as a virial expansion $f_{b}^{e x}(\phi)=$ $\sum_{i=2}^{\infty} c_{i} \phi^{i}$, with coefficients $c_{i}$ that depend on the temperature. Alternatively one may approximate, e.g., by assuming a lattice-gas free energy

$$
f_{b}^{e x}=\frac{k T}{l^{3}}[\phi+(1-\phi) \ln (1-\phi)]-\frac{b_{b}}{2} \phi^{2},
$$

where $b_{b}>0$ is an intersurfactant molecule attraction strength parameter, or instead one could assume $f_{b}^{e x}(\phi)=f_{c s}(\phi)-b_{b} \phi^{2} / 2$, where $f_{c s}(\phi)$ is the Carnahan-Starling approximation for the hard-sphere excess free energy [52].

\section{B. Pressures, chemical potentials, and surface stress}

The expression for pressure $p=\delta \mathcal{F} / \delta h$ is obtained by calculating the variation of Eq. (10) with respect to $h$ for fixed $\widetilde{\Gamma}$ and $\psi$. Thereby, the variation of $\mathcal{F}_{\mathrm{s}}$ depends on the surface metric and uses the relations

$$
\delta a=a a^{\alpha \beta} \delta a_{\alpha \beta}=-a a_{\alpha \beta} \delta a^{\alpha \beta}, \quad u \delta a_{\alpha \beta}=u \delta\left(\partial_{\alpha} h \partial_{\beta} h\right)=-\left[\partial_{\alpha}\left(u \partial_{\beta} h\right)+\partial_{\beta}\left(u \partial_{\alpha} h\right)\right] \delta h,
$$

where $u$ is an arbitrary function of the surface coordinates. Also note that $a^{\alpha \beta} a_{\alpha \beta}=\delta_{\alpha}^{\alpha}=2$. As mentioned above, $\Gamma$ changes with surface extension or contraction, so before the variation of $\mathcal{F}_{\mathrm{s}}$ is computed one needs to replace $\Gamma=\widetilde{\Gamma} / \sqrt{a}$, where $\widetilde{\Gamma}$ is a reference surfactant coverage of a planar interface or coverage per substrate area [29]. Similarly, one must replace $\phi \rightarrow \psi / h$ before the variation of $\mathcal{F}_{\mathrm{b}}$ is computed [27]. This yields

$$
\begin{gathered}
p=\frac{\delta \mathcal{F}}{\delta h}=-\partial_{\alpha}\left(\sqrt{a} \sigma^{\alpha \beta} \partial_{\beta} h\right)-\Pi(h)+p_{\mathrm{b}}, \\
p_{\mathrm{b}}=\frac{\delta \mathcal{F}_{\mathrm{b}}}{\delta h}=-p_{\text {osm }}+\kappa\left[\frac{1}{2}|\nabla \phi|^{2}+\frac{\phi}{h} \nabla \cdot(h \nabla \phi)\right], \quad p_{\text {osm }}=\phi f^{\prime}(\phi)-f(\phi),
\end{gathered}
$$

where $p_{\text {osm }}$ is the bulk osmotic pressure. With solely the ideal-gas (entropic) terms in Eq. (15), it becomes $p_{\text {osm }}=k T \phi / l^{3}$. Note too that $\nabla$ is the $2 \mathrm{D}$ gradient operator and $\nabla^{2}$ is the $2 \mathrm{D}$ Laplacian. The second term in Eq. (18) is the disjoining pressure, while the first term contains the interfacial stress

$$
\begin{aligned}
\sigma^{\alpha \beta}=\frac{\delta \mathcal{F}_{\mathrm{s}}}{\delta a_{\alpha \beta}}= & \frac{1}{2} a^{\alpha \beta}\left[f_{\mathrm{s}}(\Gamma)-\Gamma f_{\mathrm{s}}^{\prime}(\Gamma)\right]-\frac{\kappa_{\mathrm{s}}}{4} a^{\alpha \beta} a^{\gamma \delta} \partial_{\gamma} \Gamma \partial_{\delta} \Gamma \\
& +\frac{\kappa_{\mathrm{s}} \Gamma}{4} a^{\alpha \beta}\left[\partial_{\gamma}\left(\sqrt{a} a^{\gamma \delta} \partial_{\delta} \Gamma\right)+\partial_{\delta}\left(\sqrt{a} a^{\gamma \delta} \partial_{\gamma} \Gamma\right)\right] .
\end{aligned}
$$

In particular, the standard surface tension is defined as

$$
\gamma(\Gamma)=a_{\alpha \beta}\left(\sigma^{\alpha \beta}\right)_{\kappa_{\mathrm{s}} \rightarrow 0}=f_{\mathrm{s}}(\Gamma)-\Gamma f_{\mathrm{s}}^{\prime}(\Gamma) .
$$

The function $f_{\mathrm{s}}$ in Eq. (13) with Eq. (14) for $b=0$ results then in what is sometimes called the Langmuir equation of state [53,54] or the Von Szyckowski equation [55]

$$
\gamma=\gamma_{0}+\frac{k T}{l_{\mathrm{s}}^{2}} \ln (1-\Gamma),
$$

i.e., for $\Gamma \ll 1$ one has $\gamma \approx \gamma_{0}-k T \Gamma / l_{\mathrm{s}}^{2}=\gamma_{0}-\gamma_{\Gamma} \Gamma$, where we introduced the Marangoni coefficient $\gamma_{\Gamma}=k T / l_{\mathrm{s}}^{2}$ for the resulting linear solutal Marangoni effect. Note that with $b \neq 0$ in Eq. (14) one obtains the Frumkin equation of state as given in Ref. [53] and further discussed below in Sec. III E 2.

The surface chemical potential $\mu_{\mathrm{s}}$ is obtained by varying Eq. (10) with respect to $\widetilde{\Gamma}$ :

$$
\mu_{\mathrm{s}}=\frac{\delta \mathcal{F}}{\delta \widetilde{\Gamma}}=\frac{d f_{\mathrm{s}}}{d \Gamma}-\frac{\kappa_{\mathrm{s}}}{2}\left[\partial_{\alpha}\left(a^{\alpha \beta} \partial_{\beta} \Gamma\right)+\partial_{\beta}\left(a^{\alpha \beta} \partial_{\alpha} \Gamma\right)\right]
$$


Finally, the bulk chemical potential is ${ }^{5}$

$$
\mu=\frac{\delta \mathcal{F}}{\delta \psi}=f^{\prime}(\phi)-\kappa h^{-1} \nabla \cdot(h \nabla \phi) .
$$

The mechanical interaction between the surfactant layer and the bulk liquid is carried by the balance of the interfacial stress and the viscous stress in the bulk fluid proportional to the normal derivative of the velocity $v^{\alpha}$ tangential to the interface and the bulk viscosity $\eta$ :

$$
\sigma_{; \beta}^{\alpha \beta}=\eta v_{; n}^{\alpha},
$$

where the semicolon denotes the covariant derivative necessary when vectors defined on a curved interface are involved. This equation reduces to the commonly used tangential stress balance including the Marangoni force when the rigidity $\kappa_{\mathrm{s}}$ is neglected.

\section{Thin-film hydrodynamics}

The above general expressions for the surface stress and pressure can be simplified in the case when the curvature and inclination are small so that the long-wave or lubrication approximation can be made. To this end, we scale $\partial_{\alpha} \sim O(\epsilon), v^{\alpha} \sim O(\epsilon)$, and $\partial_{t} \sim O\left(\epsilon^{2}\right)$ and retain terms up to the lowest relevant order in $\epsilon \ll 1$. With this scaling, $a_{\alpha \beta}$ differs from the Cartesian surface metric $\delta_{\alpha \beta}$ by $O\left(\epsilon^{2}\right)$, so $a_{\alpha \beta}=\delta_{\alpha \beta}+\epsilon^{2} \partial_{\alpha} h \partial_{\beta} h$ and its inverse is, to leading order, $a^{\alpha \beta}=\delta_{\alpha \beta}-\epsilon^{2} \partial_{\alpha} h \partial_{\beta} h$. Then the above expressions can be rewritten using Cartesian coordinates $x_{\alpha}$ spanning the plane of the substrate, whereby the distinction between covariant and contravariant tensors disappears (so that all indices can be written as subscripts) and covariant derivatives are replaced by usual partial derivatives. Retaining the leading-order terms only, Eqs. (18)-(23) become

$$
\begin{gathered}
p=\frac{\delta \mathcal{F}}{\delta h}=-\nabla \cdot\left[\left(\gamma_{0}-p_{\mathrm{s}}\right) \nabla h\right]-\Pi(h)+p_{\mathrm{b}}, \\
p_{\mathrm{s}}=\gamma_{0}-\gamma(\Gamma)-\kappa_{\mathrm{s}}\left(\Gamma \nabla^{2} \Gamma-\frac{1}{2}|\nabla \Gamma|^{2}\right), \\
\mu_{\mathrm{s}}=\frac{\delta \mathcal{F}}{\delta \widetilde{\Gamma}}=f_{\mathrm{s}}^{\prime}(\Gamma)-\kappa_{\mathrm{s}} \nabla^{2} \Gamma,
\end{gathered}
$$

where we have used $\sigma_{\alpha \beta}=\delta_{\alpha \beta}\left(\gamma_{0}-p_{\mathrm{s}}\right)$ and $p_{\mathrm{s}}$ is the surface pressure that captures the difference between reference surface tension without surfactant $\gamma_{0}$ and the full concentration-dependent expression (including rigidity). Further, $p_{\mathrm{b}}$ and $p_{\mathrm{osm}}$ remain as in Eq. (19), while $\mu$ is still given by Eq. (24).

The bulk flow field is computed by solving the modified Stokes equation, also called the momentum equation of model $\mathrm{H}[56,57]$. Its relevant components are parallel to the substrate plane

$$
\eta \mathbf{v}^{\prime \prime}(z)=\nabla p+\phi \nabla \mu
$$

where $\mathbf{v}$ is the $2 \mathrm{D}$ vector of the velocities parallel to the substrate plane. An alternative form of Eq. (29) can be obtained using the relation

$$
\nabla p_{\mathrm{b}}=f^{\prime \prime}(\phi) \nabla \phi-\kappa \nabla\left[\frac{1}{2}|\nabla \phi|^{2}+\frac{\phi}{h} \nabla \cdot(h \nabla \phi)\right]=-\phi \nabla \mu+\frac{\kappa}{h} \nabla\left(h|\nabla \phi|^{2}\right),
$$

\footnotetext{
${ }^{5}$ Note that, formally, since $\psi$ is an effective layer thickness, $\mu$ is also a pressure, namely the partial pressure of the solute.
} 
which reduces the right-hand side of the Stokes equation (29) to

$$
\nabla\left(p-p_{\mathrm{b}}\right)+\nabla p_{\mathrm{b}}+\phi \nabla \mu=\nabla \widehat{p}+\frac{\kappa}{h} \nabla\left(h|\nabla \phi|^{2}\right),
$$

where $\widehat{p}$ is the effective pressure excluding $p_{\mathrm{b}}$. This shows that osmotic pressure $p_{\text {osm }}$ does not affect hydrodynamic flow (as $\nabla p_{\text {osm }}=\phi \nabla \mu$ ), while the contribution of the bulk rigidity is expressed by the last term in the above relation.

Solving Eq. (29) in the lubrication approximation with the no-slip boundary condition at the substrate plane $z=0$ and the momentum balance condition (25) at the interface $z=h$ yields

$$
\mathbf{v}=-\frac{z}{\eta}\left[\nabla p_{\mathrm{s}}+\left(h-\frac{z}{2}\right)(\nabla p+\phi \nabla \mu)\right] .
$$

Integrated over the local film thickness, this leads to the convective fluid flux

$$
\mathbf{J}_{\text {conv }}=\int_{0}^{h} \mathbf{v} d z=-\frac{h^{2}}{2 \eta} \nabla p_{\mathrm{s}}-\frac{h^{3}}{3 \eta}(\nabla p+\phi \nabla \mu)
$$

and the interfacial velocity $\mathbf{v}_{\mathrm{s}}=\mathbf{v}(h)$. Then the volume conservation condition

$$
\partial_{t} h=-\nabla \cdot \mathbf{J}_{\text {conv }}
$$

results, to leading order, in the evolution equation of the film thickness

$$
\partial_{t} h=\nabla \cdot\left[\frac{h^{3}}{3 \eta}(\nabla p+\phi \nabla \mu)+\frac{h^{2}}{2 \eta} \nabla p_{\mathrm{s}}\right]-J_{\mathrm{ev}}(h, \Gamma, \phi),
$$

where we now incorporated the evaporation flux $J_{\mathrm{ev}}$. The leading-order equations expressing the surface and bulk surfactant conservation laws are

$$
\begin{aligned}
\partial_{t} \Gamma & =\nabla \cdot\left(\frac{h^{2}}{2 \eta} \Gamma(\nabla p+\phi \nabla \mu)+\frac{h}{\eta} \Gamma \nabla p_{\mathrm{s}}+M_{\mathrm{s}}(\Gamma) \nabla \mu_{\mathrm{s}}\right)+\widetilde{J}_{\mathrm{ad}}(\Gamma, \phi), \\
\partial_{t} \psi & =\nabla \cdot\left(\frac{h^{2}}{3 \eta} \psi(\nabla p+\phi \nabla \mu)+\frac{h}{2 \eta} \psi \nabla p_{\mathrm{s}}+h M(\phi) \nabla \mu\right)-J_{\mathrm{ad}}(\Gamma, \phi),
\end{aligned}
$$

where $M_{\mathrm{s}}(\Gamma)$ and $M(\phi)$ are general surface and bulk mobility functions and $\tilde{J}_{\mathrm{ad}}=J_{\mathrm{ad}} / l_{\mathrm{s}}$ is the net surfactant adsorption flux; surface distortions contribute to Eq. (36) as $O\left(\epsilon^{2}\right)$ terms only. In the dilute limit, the mobilities can be expressed as

$$
M_{\mathrm{s}}(\Gamma)=\frac{D_{\mathrm{s}} l_{\mathrm{s}}^{2} \Gamma}{k T}, \quad M(\phi)=\frac{D l^{3} \phi}{k T},
$$

where $D_{\mathrm{s}}$ and $D$ are surface and bulk diffusivities, respectively. The lengths in the diffusion terms are introduced for convenience. They ensure that the diffusivities $D$ have the dimension (length) $)^{2} /($ time), as usual for diffusion constants. The conserved dynamics in Eqs. (36) and (37) have the form of conservation laws

$$
\begin{gathered}
\partial_{t} \Gamma=-\nabla \cdot\left(\Gamma \mathbf{v}_{\mathrm{s}}+\mathbf{J}_{\text {diff }}^{\Gamma}\right), \\
\partial_{t}(\phi h)=-\nabla \cdot\left(\phi \mathbf{J}_{\text {conv }}+\mathbf{J}_{\text {diff }}^{\phi}\right),
\end{gathered}
$$

respectively. We also take into account the relation

$$
\nabla p_{\mathrm{s}}=\Gamma f_{\mathrm{s}}^{\prime \prime}(\Gamma) \nabla \Gamma+\kappa_{\mathrm{s}} \nabla\left(\Gamma \nabla^{2} \Gamma-\frac{1}{2}|\nabla \Gamma|^{2}\right)=\Gamma \nabla f_{\mathrm{s}}^{\prime}(\Gamma)-\kappa_{\mathrm{s}} \Gamma \nabla \nabla^{2} \Gamma=\Gamma \nabla \mu_{\mathrm{s}},
$$

which allows us to replace the gradient of the surface pressure in Eq. (35) by $\Gamma \nabla \mu_{\mathrm{s}}$. 


\section{Gradient dynamics formulation}

Equations (36) and (37) can be now presented in the general gradient dynamics form (2) with $a, b=1,2,3$ for three fields as

$$
\begin{aligned}
\partial_{t} h & =\nabla \cdot\left(Q_{h h} \nabla \frac{\delta \mathcal{F}}{\delta h}+Q_{h \Gamma} \nabla \frac{\delta \mathcal{F}}{\delta \widetilde{\Gamma}}+Q_{h \psi} \nabla \frac{\delta \mathcal{F}}{\delta \psi}\right)-\beta_{\text {evap }}\left(\frac{\delta \mathcal{F}}{\delta h}-p_{\text {vap }}\right), \\
\partial_{t} \widetilde{\Gamma} & =\nabla \cdot\left(Q_{\Gamma h} \nabla \frac{\delta \mathcal{F}}{\delta h}+Q_{\Gamma \Gamma} \nabla \frac{\delta \mathcal{F}}{\delta \widetilde{\Gamma}}+Q_{\Gamma \psi} \nabla \frac{\delta \mathcal{F}}{\delta \psi}\right)-\beta_{\psi \Gamma}\left(\frac{1}{l_{\mathrm{s}}} \frac{\delta \mathcal{F}}{\delta \widetilde{\Gamma}}-\frac{\delta \mathcal{F}}{\delta \psi}\right), \\
\partial_{t} \psi & =\nabla \cdot\left(Q_{\psi h} \nabla \frac{\delta \mathcal{F}}{\delta h}+Q_{\psi \Gamma} \nabla \frac{\delta \mathcal{F}}{\delta \widetilde{\Gamma}}+Q_{\psi \psi} \nabla \frac{\delta \mathcal{F}}{\delta \psi}\right)-\beta_{\psi \Gamma}\left(l_{\mathrm{s}} \frac{\delta \mathcal{F}}{\delta \psi}-\frac{\delta \mathcal{F}}{\delta \widetilde{\Gamma}}\right),
\end{aligned}
$$

where on the left-hand side we use $\widetilde{\Gamma}$ for consistency (the difference to $\Gamma$ being of higher order). The mobility matrix for the conserved dynamics reads

$$
\mathbf{Q}^{\mathrm{c}}=\left(\begin{array}{ccc}
Q_{h h}^{\mathrm{c}} & Q_{h \Gamma}^{\mathrm{c}} & Q_{h \psi}^{\mathrm{c}} \\
Q_{\Gamma h}^{\mathrm{c}} & Q_{\Gamma \Gamma}^{\mathrm{c}} & Q_{\Gamma \psi}^{\mathrm{c}} \\
Q_{\psi h}^{\mathrm{c}} & Q_{\psi \Gamma}^{\mathrm{c}} & Q_{\psi \psi}^{\mathrm{c}}
\end{array}\right)=\left(\begin{array}{ccc}
\frac{h^{3}}{3 \eta} & \frac{h^{2} \Gamma}{2 \eta} & \frac{h^{2} \psi}{3 \eta} \\
\frac{h^{2} \Gamma}{2 \eta} & \frac{h \Gamma^{2}}{\eta}+M_{\mathrm{s}}(\Gamma) & \frac{h \psi \Gamma}{2 \eta} \\
\frac{h^{2} \psi}{3 \eta} & \frac{h \psi \Gamma}{2 \eta} & \frac{h \psi^{2}}{3 \eta}+h M(\phi)
\end{array}\right) .
$$

Note that $\mathbf{Q}^{\mathrm{c}}$ is symmetric and positive definite, corresponding to Onsager relations between the fluxes and positive entropy production, respectively. ${ }^{6}$ Also the mobility matrix

$$
\mathbf{Q}^{\mathrm{nc}}=\left(\begin{array}{lll}
Q_{h h}^{\mathrm{nc}} & Q_{h \Gamma}^{\mathrm{nc}} & Q_{h \psi}^{\mathrm{nc}} \\
Q_{\Gamma h}^{\mathrm{nc}} & Q_{\Gamma \Gamma}^{\mathrm{nc}} & Q_{\Gamma \psi}^{\mathrm{nc}} \\
Q_{\psi h}^{\mathrm{nc}} & Q_{\psi \Gamma}^{\mathrm{nc}} & Q_{\psi \psi}^{\mathrm{nc}}
\end{array}\right)=\left(\begin{array}{ccc}
\beta_{\mathrm{evap}} & 0 & 0 \\
0 & \frac{\beta_{\psi \Gamma}}{l_{\mathrm{s}}} & -\beta_{\psi \Gamma} \\
0 & -\beta_{\psi \Gamma} & l_{\mathrm{s}} \beta_{\psi \Gamma}
\end{array}\right)
$$

for the nonconserved dynamics is symmetric and positive definite. Note that the mobility functions that involve $\Gamma$ have a dimension different from the other terms; the same applies to the variations. However, the overall contributions to the respective fluxes have of course the same dimensions.

The final nonconserved terms in Eqs. (42)-(44) correspond to $-J_{\mathrm{ev}}, \widetilde{J}_{\mathrm{ad}}=J_{\mathrm{ad}} / l_{\mathrm{s}}$, and $-J_{\mathrm{ad}}$, respectively. We discuss below in Sec. IIIE 2 that in the limit of a flat surface and without rigidity terms they give exactly the expressions for adsorption or desorption most often derived in the literature from kinetic considerations [58,59]. However, in contrast to these considerations, our formulation also naturally captures the influence of surface modulations and rigidity effects.

Comparing the three conserved fluxes in Eqs. (42)-(44) to the conservation laws (34), (39), and (40), one notes that only $Q_{1}^{\mathrm{c}}=Q_{h h}^{\mathrm{c}}, Q_{2}^{\mathrm{c}}=Q_{h \Gamma}^{\mathrm{c}}$, and $Q_{3}^{\mathrm{c}}=Q_{\Gamma \Gamma}^{\mathrm{c}}$ are independent; the other mobility functions can be derived from the relation between $\mathbf{J}_{\text {conv }}$ and $\phi \mathbf{J}_{\text {conv }}$, i.e., the mobility matrix is

$$
\mathbf{Q}^{\mathrm{c}}=\left(\begin{array}{ccc}
Q_{1}^{\mathrm{c}} & Q_{2}^{\mathrm{c}} & \phi Q_{1}^{\mathrm{c}} \\
Q_{2}^{\mathrm{c}} & Q_{3}^{\mathrm{c}} & \phi Q_{2}^{\mathrm{c}} \\
\phi Q_{1}^{\mathrm{c}} & \phi Q_{2}^{\mathrm{c}} & \phi^{2} Q_{1}^{\mathrm{c}}
\end{array}\right)
$$

This structure ensures that for any $f(\phi)$ the osmotic pressure in the bulk film $p_{\text {osm }}$ does not contribute to the convective flux $J_{\text {conv }}$. However, it does have an influence on evaporation (see Sec. III E). Without slip at the solid substrate, one has $Q_{1}^{\mathrm{c}}=h^{3} / 3 \eta, Q_{2}^{\mathrm{c}}=\Gamma h^{2} / 2 \eta$, and $Q_{3}^{\mathrm{c}}=\Gamma^{2} h / \eta$, but slip can be easily incorporated.

\footnotetext{
${ }^{6}$ Note that the expression of the rate of free-energy dissipation through bulk and surface diffusive fluxes and the liquid velocity is actually simple and short. However, the coupling between the three fields $h, \widetilde{\Gamma}$, and $\psi$ results in the complicated form of the mobility matrix $\mathbf{Q}^{\mathrm{c}}$.
} 


\section{E. Nonconserved fluxes}

The general gradient dynamics form in Eqs. (42)-(44) incorporates conserved and nonconserved fluxes. The considered nonconserved fluxes include an evaporation-condensation flux $J_{\mathrm{ev}}$ that only enters the equation for the film height (42) and an adsorption-desorption flux $J_{\text {ad }}$ that enters the equations for the bulk and surface concentrations (43) and (44). If the respective fluxes are zero, the exchange processes are at equilibrium, i.e., the evaporation and condensation of the solvent balance as well as adsorption and desorption of the solute. In the following we discuss the fluxes individually.

\section{Evaporation and condensation}

Assuming that the solute does not influence the film height, the evaporation flux is given by

$$
J_{\mathrm{ev}}(h, \Gamma, \phi)=\beta_{\text {evap }}\left(\frac{\delta \mathcal{F}}{\delta h}-p_{\text {vap }}\right) .
$$

With (26) and (19) this becomes

$$
J_{\mathrm{ev}}(h, \Gamma, \phi)=\beta_{\text {evap }}\left(\nabla \cdot\left(p_{\mathrm{s}} \nabla h\right)-\Pi(h)-p_{\mathrm{osm}}-\kappa\left[\frac{1}{2}|\nabla \phi|^{2}+\frac{\phi}{h} \nabla \cdot(h \nabla \phi)\right]-p_{\text {vap }}\right),
$$

where as before $p_{\text {osm }}=\phi f^{\prime}(\phi)-f(\phi)$ and $p_{\text {vap }}$ is the partial vapor pressure in the ambient air. Besides the known Kelvin effect [first term on the right-hand side, here with the full dependence $\left.p_{\mathrm{s}}(\Gamma)\right][60]$, also wettability (second term on the right-hand side) and osmotic pressure (third term) influence evaporation, as does the bulk rigidity (fourth term). Normally, even on mesoscopic scales, the dominant term is that involving the vapor pressure (fifth term) and this term largely controls the evaporation rate (see Ref. [15] for further discussion on this). However, the other terms do matter close to contact lines, for nanodroplets, and at diffuse interfaces of dense and dilute phases. Note that such thermodynamically consistent relations for $J_{\mathrm{ev}}$ are also obtained for all the model extensions discussed below in Sec. IV. Also note that the rate $\beta_{\text {evap }}$ is not necessarily constant. It may depend on film height, e.g., $\beta_{\text {evap }}=E /(K+h)$ when incorporating effects of latent heat [61-63] (see [15] for more details).

Problems may arise in the limit of very high bulk concentrations of the solute, since the physical film height can then be virtually identical to the effective solute height in contradiction to the model assumption that the effective solute height is small as compared to the effective solvent height that is identified with the film height. This issue may be resolved through a solvent-solute symmetric model as proposed in Ref. [36] in the two-field case. This case of high solute concentrations is not pursued here.

\section{Adsorption and desorption}

Besides evaporation, the nonconserved part of the gradient dynamics (42)-(44) also describes the dynamics of exchange of surfactant molecules between the liquid bulk and the free surface. When $\widetilde{J}_{\mathrm{ad}}>0$ this corresponds to an adsorption flux of molecules attaching to the free surface, while when $\widetilde{J}_{\text {ad }}<0$ there is desorption from the free surface, i.e., it is an influx into the bulk. Overall, the exchange between the bulk and the free surface is mass conserving, i.e., it suffices to discuss $\widetilde{J}_{\text {ad }}$, and then $J_{\mathrm{ad}}=l_{\mathrm{s}} \widetilde{J}_{\mathrm{ad}}$. Within the gradient dynamics it is given by

$$
\begin{aligned}
\widetilde{J}_{\mathrm{ad}}(h, \Gamma, \phi) & =\beta_{\psi \Gamma}\left(\frac{\delta \mathcal{F}}{\delta \psi}-\frac{1}{l_{\mathrm{s}}} \frac{\delta \mathcal{F}}{\delta \widetilde{\Gamma}}\right) \\
& =\beta_{\psi \Gamma}\left(\mu-\frac{1}{l_{\mathrm{s}}} \mu_{\mathrm{s}}\right) \\
& =\beta_{\psi \Gamma}\left[\frac{d f}{d \phi}-\kappa h^{-1} \nabla \cdot(h \nabla \phi)-\frac{1}{l_{\mathrm{s}}}\left(\frac{d f_{\mathrm{s}}}{d \Gamma}-\kappa_{\mathrm{s}} \nabla^{2} \Gamma\right)\right],
\end{aligned}
$$


where we have used Eqs. (24) and (28). Note that the bulk rigidity $(\kappa \neq 0)$ introduces an explicit film height dependence. Without rigidity influences $\left(\kappa, \kappa_{\mathrm{s}}=0\right)$, the flux is $\widetilde{J}_{\mathrm{ad}}=\beta_{\psi \Gamma}\left[\frac{d f}{d \phi}-\frac{1}{l_{\mathrm{s}}} \frac{d f_{\mathrm{s}}}{d \Gamma}\right]$ and one may now consider several particular cases.

In the dilute limit for the bulk concentration $\phi$ we have $f_{b}^{e x}=0$ and Eq. (15) becomes

$$
f(\phi)=\frac{k T}{l^{3}}[\phi(\ln \phi-1)] .
$$

This implies that when solely entropic surface packing effects are included in $f_{\mathrm{s}}(\Gamma)$, i.e., Eqs. (13) and (14) with the intermolecular attraction parameter $b=0$, we obtain

$$
\widetilde{J}_{\mathrm{ad}}=\beta_{\psi \Gamma} \frac{k T}{l_{\mathrm{s}}^{3}} \ln \frac{(1-\Gamma) \phi}{\Gamma},
$$

where we also assume $l=l_{\mathrm{s}}\left(\right.$ otherwise $\left.\phi \rightarrow \phi^{l_{\mathrm{s}}^{3} / l^{3}}\right){ }^{7}$

In many cases, the surfactant isotherms that relate equilibrium surface concentration $\Gamma_{\mathrm{eq}}$ and equilibrium bulk concentration $\phi_{\text {eq }}$ are introduced based on kinetic arguments of equal desorption and adsorption fluxes (see, e.g., Refs. [58,59]). However, the isotherm is an equilibrium property and may be directly obtained from the free energy. In the present context, one has at equilibrium $\widetilde{J}_{\mathrm{ad}}=0$, i.e., $\phi_{\mathrm{eq}}=\Gamma_{\text {eq }} /\left(1-\Gamma_{\text {eq }}\right)$ or $\Gamma_{\text {eq }}=\phi_{\text {eq }} /\left(1+\phi_{\text {eq }}\right)$ corresponding to the Langmuir adsorption isotherm [58]. To obtain the kinetics when the system is out of but still close to equilibrium we expand the logarithm in Eq. (54) about the equilibrium state and obtain

$$
\widetilde{J}_{\mathrm{ad}} \approx \beta_{\psi \Gamma} \frac{k T}{\Gamma l_{\mathrm{s}}^{3}}[(1-\Gamma) \phi-\Gamma] .
$$

This expression for the effective adsorption flux (adsorption minus desorption) agrees for $\beta_{\psi \Gamma}=\tilde{M} \Gamma$ up to normalization factors with Eq. (6) of Ref. [53] that result from kinetic considerations.

One may also go beyond purely entropic interactions, e.g., by using Eq. (14) or other forms of $f_{\mathrm{s}}^{e x}(\Gamma)$. With $b>0$ in Eq. (14) one introduces a simple attraction between surfactant molecules at the free surface. Then

$$
\widetilde{J}_{\mathrm{ad}}=\beta_{\psi \Gamma} \frac{k T}{l_{\mathrm{s}}^{3}} \ln \frac{(1-\Gamma) \phi^{l_{\mathrm{s}}^{3} / l^{3}}}{\Gamma}+\beta_{\psi \Gamma} \frac{b \Gamma}{l_{\mathrm{s}}},
$$

where this time we retain the general $l \neq l_{\mathrm{s}}$.

At equilibrium $\widetilde{J}_{\mathrm{ad}}=0$, i.e.,

$$
\phi_{\mathrm{eq}}=\left(\frac{\Gamma_{\mathrm{eq}}}{1-\Gamma_{\mathrm{eq}}}\right)^{\left(l / l_{\mathrm{s}}\right)^{3}} e^{-\tilde{b} \Gamma_{\mathrm{eq}}},
$$

where $\tilde{b}=b l^{3} / k T l_{\mathrm{s}}$, or in an implicit form

$$
\Gamma_{\mathrm{eq}}=\frac{\phi_{\mathrm{eq}}^{\left(l_{\mathrm{s}} / l\right)^{3}} e^{\tilde{b}\left(l_{\mathrm{s}} / l\right)^{3} \Gamma_{\mathrm{eq}}}}{1+\phi_{\mathrm{eq}}^{\left(l_{\mathrm{s}} / l\right)^{3}} e^{\tilde{b}\left(l_{\mathrm{s}} / l\right)^{3} \Gamma_{\mathrm{eq}}}} .
$$

Both are common in the literature [65]; in particular, for $l=l_{\mathrm{s}}$ they are known as the Frumkin isotherm (see [58], Chap. 2.N)

$$
\phi_{\mathrm{eq}}=\left(\frac{\Gamma_{\mathrm{eq}}}{1-\Gamma_{\mathrm{eq}}}\right) e^{-\tilde{b} \Gamma_{\mathrm{eq}}}
$$

\footnotetext{
${ }^{7}$ An expression identical to (54) is given in Sec. 2.3 of [64], where a free-energy approach is followed to study the kinetics of surfactant adsorption [set $\beta=0$ in their Eq. (2.14) to recover the purely entropic case]. For full agreement with [64] one needs $\beta_{\psi \Gamma}=\widetilde{M} \phi$, where $\widetilde{M}$ is a molecular mobility. The approximation discussed next makes it likely that there is actually a typographical error in Ref. [64] and it should read $\beta_{\psi \Gamma}=\widetilde{M} \Gamma$.
} 
or

$$
\Gamma_{\mathrm{eq}}=\frac{\phi_{\mathrm{eq}}}{e^{-\tilde{b} \Gamma_{\mathrm{eq}}}+\phi_{\mathrm{eq}}} .
$$

The kinetic adsorption equation given in Ref. [66] is obtained by expanding (56) about this equilibrium state (59). The linearized flux is

$$
\widetilde{J}_{\mathrm{ad}}=\beta_{\psi \Gamma} \frac{k T}{l^{3} \Gamma} e^{\tilde{b} \Gamma}\left[(1-\Gamma) \phi-\Gamma e^{-\tilde{b} \Gamma}\right],
$$

which has the same form as Eq. (16) of Ref. [66] and implies certain $\Gamma$ dependences of their mobilities $\alpha$ and $\beta$ or of our mobility $\beta_{\psi \Gamma}$. Note that the case of adhesion (their $K<0$ ) here corresponds to $\tilde{b}>0$.

The expression in Eq. (49), which is linear in the thermodynamic potentials (variations of $\mathcal{F}$ ), must be linearized about the equilibrium state $\widetilde{J}_{\text {ad }}=0$ to obtain the expressions obtained in the literature based on kinetic considerations. This may imply that these kinetic considerations only capture a linearized picture of the process. Alternatively, one may introduce expressions such as $(\phi-\Gamma) /(\ln \phi-\ln \Gamma)$ into the mobility $\beta_{\psi \Gamma}$ as proposed in Ref. [67] in the context of gradient dynamics formulations of reaction-diffusion dynamics. However, for the more complicated free energies discussed here this seems inadequate. Another option is to go beyond linear nonequilibrium thermodynamics, i.e., beyond the expression linear in the thermodynamic potentials in Eq. (49). For activated processes, activation barriers have to be overcome and Arrhenius-type exponential factors may be appropriate. For instance, an adsorption flux

$$
\widetilde{J}_{\mathrm{ad}}(h, \Gamma, \phi)=\hat{\beta}_{\psi \Gamma}\left(\exp \left[-\frac{a^{3}}{k T} \frac{\delta \mathcal{F}}{\delta \psi}+\frac{a^{3}}{k T l_{\mathrm{s}}} \frac{\delta \mathcal{F}}{\delta \widetilde{\Gamma}}\right]-1\right)
$$

( $a$ is a microscopic length scale) with appropriately defined mobility $\hat{\beta}_{\psi \Gamma}$ results in the same expressions for the flux as obtained via kinetic considerations.

We end this section with a side remark on the general adsorption isotherm. Using the standard definition of the surface tension given in Eq. (21), we obtain

$$
d \gamma=-\Gamma_{\mathrm{eq}} f_{\mathrm{s}}^{\prime \prime} d \Gamma_{\mathrm{eq}}=-\Gamma_{\mathrm{eq}} f_{\mathrm{s}}^{\prime \prime} \frac{d \Gamma_{\mathrm{eq}}}{d\left(\ln \phi_{\mathrm{eq}}\right)} d\left(\ln \phi_{\mathrm{eq}}\right) .
$$

In the dilute limit of the bulk surfactant concentration, i.e., for $f(\phi)=\frac{k T}{l^{3}} \phi(\ln \phi-1)$, the adsorption isotherm is $\left(k T l_{\mathrm{s}} / l^{3}\right) \ln \phi_{\mathrm{eq}}=f_{\mathrm{s}}^{\prime}$, i.e., $d\left(\ln \phi_{\mathrm{eq}}\right) / d \Gamma_{\mathrm{eq}}=\left(l^{3} / k T l_{\mathrm{s}}\right) f_{\mathrm{s}}^{\prime \prime}$, implying that the Gibbs adsorption isotherm

$$
d \gamma=-\frac{k T l_{\mathrm{s}}}{l^{3}} \Gamma_{\mathrm{eq}} d\left(\ln \phi_{\mathrm{eq}}\right)
$$

is valid for any form of $f_{\mathrm{s}}^{\prime \prime}(\Gamma)$. However, this is not the case for more complicated expressions for $f(\phi)$ or indeed when rigidity effects are included. Then Eq. (52) with $\widetilde{J}_{\mathrm{ad}}=0$ provides a general relation valid for heterogeneous equilibria.

\section{SOLUBLE SURFACTANT: SPECIAL CASES AND EXTENSIONS}

In this section we explore further the general gradient dynamics model (42)-(44). In particular, we first show that well known hydrodynamic long-wave models are recovered as limiting cases. We then discuss extensions incorporating physical effects of interest that can be described within the present framework. 


\section{A. Hydrodynamic formulation in dilute limit}

The standard hydrodynamic long-wave model employed for thin films with a soluble surfactant that is dilute within the film and also has a low coverage at the film surface [4,5] is recovered from the general gradient dynamics form (42)-(44) for zero rigidity $\left(\kappa=0\right.$ and $\left.\kappa_{\mathrm{s}}=0\right)$ and with only the low-concentration entropic (ideal-gas) terms in the energy, i.e., neglecting the nonlinear interaction terms in the energies. Then Eqs. (13) and (15) become

$$
f_{\mathrm{s}}(\Gamma)=\gamma_{0}+\frac{k T}{l_{\mathrm{s}}^{2}} \Gamma(\ln \Gamma-1), \quad f(\phi)=\frac{k T}{l^{3}} \phi(\ln \phi-1),
$$

respectively, where $\gamma_{0}$ is a constant. The energy functional (10) in the long-wave approximation is

$$
\mathcal{F}=\int\left[h f(\phi)+f_{\mathrm{s}}(\Gamma) \xi+g(h)\right] d x d y,
$$

where $\xi=1+\frac{1}{2}(\nabla h)^{2}$. Note that in Eq. (66) one has to write $\phi=\psi / h$ and $\Gamma=\widetilde{\Gamma} / \xi$ to obtain the variations with respect to the independent fields $h, \psi$, and $\widetilde{\Gamma}$, as discussed at the beginning of Sec. III A. The variations are

$$
\begin{aligned}
p & =\frac{\delta \mathcal{F}}{\delta h}=-\partial_{x}\left[\gamma(\Gamma) \partial_{x} h\right]-\Pi(h)-\frac{k T}{l^{3}} \phi, \\
\mu_{\mathrm{s}} & =\frac{\delta \mathcal{F}}{\delta \widetilde{\Gamma}}=\frac{k T}{l_{\mathrm{s}}^{2}} \ln \Gamma, \\
\mu & =\frac{\delta \mathcal{F}}{\delta \psi}=\frac{k T}{l^{3}} \ln \phi,
\end{aligned}
$$

where $\gamma(\Gamma)=f_{\mathrm{s}}-\Gamma f_{\mathrm{s}}^{\prime}=\gamma_{0}-k T \Gamma / l_{\mathrm{s}}^{2}=\gamma_{0}-\gamma_{\Gamma} \Gamma$, i.e., purely entropic low-concentration contributions to the free-energy result in a linear equation of state. As a result, the evolution equations (42)-(46) become

$$
\begin{aligned}
\partial_{t} h=\nabla \cdot\left(\frac{h^{3}}{3 \eta} \nabla[-\nabla \cdot(\gamma \nabla h)-\Pi(h)]+\frac{\gamma_{\Gamma} h^{2}}{2 \eta} \nabla \Gamma\right)-\beta_{\text {evap }}\left(\hat{\mu}-\nabla \cdot(\gamma \nabla h)-\Pi(h)-\frac{k T}{l^{3}} \phi\right), \\
\partial_{t} \Gamma=\nabla \cdot\left[\frac{h^{2} \Gamma}{2 \eta} \nabla[-\nabla \cdot(\gamma \nabla h)-\Pi(h)]+\left(\frac{\gamma_{\Gamma} h \Gamma}{\eta}+D_{\mathrm{s}}\right) \nabla \Gamma\right]+\frac{\beta}{l}(\ln \phi-\ln \Gamma), \\
\partial_{t} \psi=\nabla \cdot\left(\frac{h^{2} \psi}{3 \eta} \nabla[-\nabla \cdot(\gamma \nabla h)-\Pi(h)]+\frac{\gamma_{\Gamma} h \psi}{2 \eta} \nabla \Gamma+D h \nabla \phi\right)-\beta(\ln \phi-\ln \Gamma),
\end{aligned}
$$

where we have assumed $l_{\mathrm{s}}=l$, used the mobility functions (38), and introduced $\beta=\beta_{\psi \Gamma} k T / l^{2}$ and

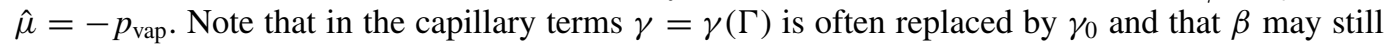
depend on the concentrations.

The model can be related to the standard hydrodynamic long-wave models for films with soluble surfactants found in the literature. In the simple case without solvent evaporation $\left(\beta_{\text {evap }}=0\right)$ and without wettability ( $\Pi=0)$, it corresponds to Eqs. (117)-(119) of the review [5] if the expression $\ln \phi-\ln \Gamma$ in our adsorption flux is replaced by the linearized $\phi-\Gamma$ as already discussed in Sec. III E 2. Equation (21) of [68] [also cf. Eqs. (4.29a)-(4.29c) of the review in Ref. [4]] further neglects all Laplace pressure contributions (equivalent to $\gamma \approx 0$, but keeping Marangoni flows) and adds permeability of the substrate for the surfactant. In Ref. [69] the case of a volatile solvent is studied for a surfactant-covered film on a heated substrate. Their Eqs. (50)-(52) add thermal Marangoni flows to our Eqs. (68)-(70) and have a linearized adsorption flux and an evaporation flux $\sim 1 /(h+K)$ that in our equation correspond to $\beta_{\text {evap }} \sim 1 /(h+K)$ and a $\hat{\mu}$ that is much larger than the other evaporation terms. 


\section{B. Mixture of liquids without surfactant}

Another important limit is the case of a liquid film of a binary mixture that consists of components that change the surface tension without forming a proper monolayer of surfactant molecules at the free surface. References [27,28] presented a two-field gradient dynamics model for the evolution of a film of a liquid binary mixture on a solid substrate that allows for the description of coupled dewetting and decomposition processes for arbitrary bulk (mixing) energies including bulk rigidity terms, capillarity, and wetting energies that may depend on the film height and concentration. The two fields are the film height $h$ and the effective solute layer height $\psi$. The model recovers, for instance, the long-wave limit of model $H$ (Navier-Stokes Cahn-Hilliard equations) as derived in Ref. [70], but also goes far beyond as it allows for a number of other systematic extensions [27,28].

However, this two-field model has an important shortcoming: In Ref. [28] it was noted that no obvious way exists to incorporate a concentration-dependent surface tension into the model without breaking the gradient dynamics structure. This implies that introducing a Marangoni flow caused by the solutal Marangoni effect into the hydrodynamic two-field thin-film model for a mixture could break the thermodynamic consistency: If one incorporates a concentration-dependent surface tension directly into the energy functional $(\gamma(\phi)$ in Eq. (1) of [28]) that only depends on the height-averaged bulk concentration $\phi$ and film height $h$, a Marangoni-like flux term is obtained, however, with the wrong prefactor in the mobility function. Therefore, the use of the model in Ref. [28] is limited to cases where surface activity can be neglected.

Here, in the context of the three-field model, this issue is resolved in the following way. We show that one may take the full gradient dynamics model for soluble surfactants introduced above in Sec. III D and consider the limit of very fast (instantaneous) adsorption and desorption. This limit corresponds to $\beta_{\psi \Gamma} \gg 1$ in Eqs. (43) and (44), implying that the nonconserved fluxes equilibrate fast. As a result, on the slower time scale of the conserved fluxes one has $J_{\text {ad }} \approx 0$ [cf. Eqs. (50)-(52)] and the surfactant concentration at the free surface is slaved to the one in the bulk film. The dependence corresponds to the equilibrium relations discussed in Sec. III E 2.

For example, in the case without rigidity one has $f^{\prime}(\phi)=f_{\mathrm{s}}^{\prime}(\Gamma) / l_{\mathrm{s}}$ and in the limit of low concentrations $\Gamma, \phi \ll 1$ and for $l_{\mathrm{s}} \approx l$ one obtains $\Gamma \approx \phi$. Then the governing equations (42)-(45) with the mobility functions (38) can be simplified by multiplying Eq. (43) by $l$ and adding it to Eq. (44). As a result, an evolution equation for $\widetilde{\psi}=\psi+l \Gamma=(h+l) \phi \approx h \phi=\psi$ is obtained where we use $h \gg l$. Dropping the tilde and approximating the mobilities according to $h \gg l$, the equation reads

$$
\partial_{t} \psi=\nabla \cdot\left[\frac{h^{2} \psi}{3 \eta} \nabla \frac{\delta F}{\delta h}+\left(\frac{\psi^{2}}{2 \eta}+\frac{D_{\mathrm{s}} l^{3} \phi}{k T}\right) \nabla \frac{\delta F}{\delta \widetilde{\Gamma}}+\left(\frac{h \psi^{2}}{3 \eta}+\frac{D l^{3} \psi}{k T}\right) \nabla \frac{\delta F}{\delta \psi}\right]
$$

The film height equation (42) becomes

$$
\partial_{t} h=\nabla \cdot\left[\frac{h^{3}}{3 \eta} \nabla \frac{\delta F}{\delta h}+\frac{h^{2} \phi}{2 \eta} \nabla \frac{\delta F}{\delta \widetilde{\Gamma}}+\frac{h^{2} \psi}{3 \eta} \nabla \frac{\delta F}{\delta \psi}\right]-\beta_{\text {evap }}\left(\frac{\delta F}{\delta h}-p_{\text {vap }}\right)
$$

As we are in the dilute limit for $f_{\mathrm{s}}$, the second term in the conserved part of (72) becomes $\gamma_{\Gamma} h^{2} \nabla \phi / 2$ with $\gamma_{\Gamma}=k T / l^{2}$ corresponding to the standard form of the Marangoni flux. The hydrodynamic form of Eq. (72) is then

$$
\partial_{t} h=\nabla \cdot\left[-\frac{h^{3}}{3 \eta} \nabla\left[\gamma_{0} \Delta h+\Pi(h)\right]+\gamma_{\Gamma} \frac{h^{2}}{2 \eta} \nabla \phi\right]
$$


while Eq. (71) becomes (again with $h \gg l$ and approximating $\gamma$ by the reference value $\gamma_{0}$ in the capillary term)

$$
\partial_{t}(\phi h)=\nabla \cdot\left[-\frac{h^{3} \phi}{3 \eta} \nabla\left[\gamma_{0} \Delta h+\Pi(h)\right]+\left(\gamma_{\Gamma} \frac{h^{2} \phi}{2 \eta}+D h\right) \nabla \phi\right]
$$

with the bulk diffusion constant $D$. Equations (73) and (74) correspond exactly to the hydrodynamic thin-film equations employed, e.g., in the study of coalescence and noncoalescence of sessile drops of mixtures in Refs. [71,72]. We emphasize that as shown here they may be derived from the full three-field gradient dynamics model in the dilute limit. Remarkably, the resulting model cannot be brought into the form of a two-field gradient dynamics. This poses the intriguing question whether there exist circumstances (consistent with the employed approximations) where the broken gradient dynamics structure can result in unphysical behavior. This merits further consideration. We finally remark that the proposed reduction from the three-field gradient dynamics model to a two-field model also works for other choices of the energies (also with rigidities); they only have to be consistent between bulk and surface.

\section{Nonlinear equation of state}

In the literature, thin-film dynamics is sometimes studied in the case of soluble surfactants with equations similar to Eqs. (68)-(70) but employing nonlinear equations of state $\gamma(\Gamma)$ (e.g., Eqs. (8)-(12) of Ref. [73]). Other examples of nonlinear equations of state in thin-film hydrodynamics are found in Refs. [74-77]. Often, the nonlinearity is incorporated into the Marangoni term and the remaining equation is left unchanged. This may lead to spurious results if the underlying gradient dynamics structure is broken. ${ }^{8}$ If instead the free-energy functional is appropriately changed one finds that Marangoni flux, diffusion, and adsorption or desorption terms all change in a consistent manner.

In the case without rigidity $\left(\kappa=\kappa_{\mathrm{s}}=0\right)$ and without evaporation the resulting equations are

$$
\begin{gathered}
\partial_{t} h=-\nabla \cdot\left[\frac{h^{3}}{3 \eta} \nabla\{\nabla \cdot[\gamma(\Gamma) \nabla h]+\Pi(h)\}+\frac{h^{2}}{2 \eta} \nabla \gamma(\Gamma)\right] \\
\partial_{t} \Gamma=-\nabla \cdot\left\{\frac{h^{2} \Gamma}{2 \eta} \nabla\{\nabla \cdot[\gamma(\Gamma) \nabla h]+\Pi(h)\}+\left[\frac{h \Gamma}{\eta}+\frac{D_{\mathrm{s}} l_{\mathrm{s}}^{2}}{k T}\right] \nabla \gamma(\Gamma)\right\}+\widetilde{J}_{\mathrm{ad}}(\Gamma, \phi), \\
\partial_{t} \psi=-\nabla \cdot\left\{\frac{h^{2} \psi}{3 \eta} \nabla\{\nabla \cdot[\gamma(\Gamma) \nabla h]+\Pi(h)\}+\frac{h}{2 \eta} \psi \nabla \gamma(\Gamma)-\frac{D l^{3} h}{k T} \nabla p_{\mathrm{osm}}(\phi)\right\}-J_{\mathrm{ad}}(\Gamma, \phi),
\end{gathered}
$$

where we used $\Gamma \nabla f_{s}^{\prime}(\Gamma)=-\nabla \gamma(\Gamma)$ and $\phi \nabla f^{\prime}(\phi)=\nabla p_{\text {osm }}$ to express surface and bulk diffusion in terms of the surface tension and osmotic pressure, respectively. For a discussion of the adsorption fluxes see Sec. III E 2 .

Nonlinear equations of state used in the literature are, for instance, the Scheludko equation of state [73-75]

$$
\gamma(\Gamma)=\frac{\gamma_{0}}{[1+\theta \Gamma]^{3}},
$$

the exponential relation $\gamma(\Gamma)=\exp (-\alpha \Gamma)$ [76], and the expression $\gamma(\Gamma)=\gamma_{0}-R T \Gamma_{\infty} \ln (1-$ $\left.\Gamma / \Gamma_{\infty}\right)$ [77]. If diffusion is expressed in the form of Fick's law $j_{\text {diff }}=\widetilde{D}(\Gamma) \nabla \Gamma$, the nonlinear diffusion constant $\widetilde{D}(\Gamma)$ should then be proportional to $d \gamma(\Gamma) / d \Gamma$, if a constant molecular diffusivity $D_{\mathrm{s}}$ is assumed [cf. Eq. (76)]. If one does not assume $\widetilde{D}(\Gamma) \sim d \gamma(\Gamma) / d \Gamma \sim-\Gamma f_{s}^{\prime \prime}(\Gamma)$, as is the case

\footnotetext{
${ }^{8}$ Normally, the equations can still be brought into the gradient dynamics form by suitably modifying the dependence of the molecular diffusivity on concentration.
} 
in all the mentioned works, then it should be realized that implicitly a certain nonlinear dependence of the molecular diffusivity on the concentration is being assumed, which may often not be justified.

\section{Concentration-dependent wettability}

The energy functional $\mathcal{F}$ described above in Sec. III A contains well separated bulk contributions $\mathcal{L}_{\mathrm{b}}$ and surface contributions $\mathcal{L}_{\mathrm{s}}$, namely, Eqs. (12) and (11), respectively. Energetic couplings (terms that depend on more than one of the independent fields) exist due to the surface metric and the introduction of the three independent fields $h, \widetilde{\Gamma}$, and $\psi$. However, the bulk free energy $f(\phi)$, surface free energy $f_{\mathrm{s}}(\Gamma)$, and wetting energy $g(h)$ may also depend on the other fields. First, we consider a concentration-dependent wetting energy.

It has been discussed several times how to incorporate such a dependence into the known hydrodynamic long-wave equations. One approach is to make the interaction constants within the Derjaguin pressure depend on the surfactant concentration (the case of insoluble surfactant) [7881]. Another approach uses a (structural) Derjaguin pressure that depends on the concentration of nanoparticles to model layering effects [82]. Reference [83] includes a concentration-dependent disjoining pressure and accounts for surfactant layers at the free surface and the solid substrate. In the bulk film dissolved surfactant molecules as well as micelles are considered. Similar extensions are made in Ref. [80] for a two-layer system with surfactant.

We argue that incorporating such concentration dependences of wetting and dewetting phenomena has to start with an amended energy functional. Then a concentration-dependent Derjaguin pressure as often introduced is one natural consequence but is not the only one. We illustrate this by replacing $g(h)$ in Eq. (12) by the general expression $g(h, \Gamma, \phi)$ for the case without rigidities $\left(\kappa=\kappa_{s}=0\right)$ but keep $f=f(\phi)$ and $f_{\mathrm{s}}=f_{\mathrm{s}}(\Gamma)$. Then the variations in the long-wave approximation are

$$
\begin{gathered}
p=\frac{\delta F}{\delta h}=f-\phi \partial_{\phi} f+\partial_{h} g-\frac{\phi}{h} \partial_{\phi} g-\nabla \cdot(\tilde{\omega} \nabla h), \\
\mu_{\mathrm{s}}=\frac{\delta F}{\delta \widetilde{\Gamma}}=\partial_{\Gamma} g+\partial_{\Gamma} f_{\mathrm{s}}, \\
\mu=\frac{\delta F}{\delta \psi}=\frac{1}{h} \partial_{\phi} g+\partial_{\phi} f,
\end{gathered}
$$

with the generalized surface tension

$$
\tilde{\omega}=f_{\mathrm{s}}-\Gamma \partial_{\Gamma} f_{\mathrm{s}}-\Gamma \partial_{\Gamma} g .
$$

Note the additional contributions that depend on $\partial_{\phi} g$ or $\partial_{\Gamma} g$ that appear in $p, \mu_{\mathrm{s}}, \mu$, and $\tilde{\omega}$. They are often missing in the literature. The full expressions for $\kappa \neq 0, \kappa_{s} \neq 0$, and general $f$ and $f_{\mathrm{s}}$ are given in Appendix B.

With Eqs. (79)-(81) the general gradient dynamics form (42)-(45) of the evolution equations becomes

$$
\begin{aligned}
\partial_{t} h=\nabla & \cdot\left(\frac{h^{3}}{3 \eta}\left[\nabla\left[\partial_{h} g-\nabla \cdot(\tilde{\omega} \nabla h)\right]-\frac{\nabla \phi}{h} \partial_{\phi} g\right]+\frac{h^{2} \Gamma}{2 \eta} \nabla\left[\partial_{\Gamma} g+\partial_{\Gamma} f_{\mathrm{s}}\right]\right)-J_{\mathrm{ev}}(h, \Gamma, \phi), \\
\partial_{t} \Gamma=\nabla & \left\{\frac{h^{2} \Gamma}{2 \eta}\left[\nabla\left[\partial_{h} g-\nabla \cdot(\tilde{\omega} \nabla h)\right]-\frac{\nabla \phi}{h} \partial_{\phi} g\right]+\left(\frac{h \Gamma^{2}}{\eta}+\frac{D_{\mathrm{s}} l_{\mathrm{s}}^{2} \Gamma}{k T}\right) \nabla\left[\partial_{\Gamma} g+\partial_{\Gamma} f_{\mathrm{s}}\right]\right\} \\
+ & \widetilde{J}_{\mathrm{ad}}(h, \Gamma, \phi), \\
\partial_{t} \psi= & \nabla \cdot\left(\frac{h^{2} \psi}{3 \eta}\left[\nabla\left[\partial_{h} g-\nabla \cdot(\tilde{\omega} \nabla h)\right]-\frac{\nabla \phi}{h} \partial_{\phi} g\right]\right. \\
& \left.+\frac{h \psi \Gamma}{2 \eta} \nabla\left[\partial_{\Gamma} g+\partial_{\Gamma} f_{\mathrm{s}}\right]+\frac{D l^{3} \psi}{k T} \nabla\left[\frac{1}{h} \partial_{\phi} g+\partial_{\phi} f\right]\right)-l_{\mathrm{s}} \widetilde{J}_{\mathrm{ad}}(h, \Gamma, \phi)
\end{aligned}
$$


The nonconserved terms are only written in summary form, but can be easily obtained with Eqs. (79)(81) from Eqs. (49) and (50).

Inspecting Eqs. (83)-(85), one notices that the above-mentioned cross-coupling terms depending on $\partial_{\phi} g$ or $\partial_{\Gamma} g$ contribute to all conserved and nonconserved fluxes. These terms are important for very thin films and in contact line regions where the free liquid-gas interface approaches the solid-liquid interface. There they contribute to diffusion, act as Marangoni-like driving terms of the convective flux, and influence adsorption and evaporation. ${ }^{9}$ For drops of mixtures, a concentration-dependent wettability might, e.g., result in a local phase decomposition in the contact line region or in a single-component wetting layer (precursor films) as observed, e.g., in experiments with polymer solutions $[84,85]$. Note that Derjaguin pressure isotherms for binary mixtures have already been discussed in Ref. [86].

\section{E. Surfactant phase transitions and mixture decomposition: Bulk and surface rigidity}

In Secs. IV B and IV C we have discussed concentration-dependent bulk energies $f(\phi)$ and surface energies $f_{\mathrm{s}}(\phi)$. If these are nonlinear and exhibit negative second derivatives, then the system is thermodynamically unstable over the corresponding concentration range. In such a case a phase decomposition in the bulk film [87] or a surfactant phase transition [88,89] may occur. Then a theoretical description needs to include rigidity effects, i.e., $\kappa \neq 0$ and/or $\kappa_{s} \neq 0$, to assign an energetic cost to strong concentration gradients. Long-wave models that include these terms were already developed for mixtures with components that are not surface active $[28,70]$ and insoluble surfactants $[29,90,91]$. In the case of constant rigidities $\kappa_{s}$ and $\kappa$, a model for soluble surfactants essentially combines the rigidity-related expressions developed in Refs. [28,29]. Therefore, here we do not explicitly write the bulky expressions. However, the variations of the energy functional in the general case are given as Eqs. (B24)-(B26) in Appendix B, so the dynamic equations can be easily obtained by introducing them into the general gradient dynamics form (42)-(45). The case of concentration-dependent rigidities may also be treated and these result in additional contributions to the variations. Finally, note that the effect of substrate-mediated condensation described in Refs. [88,89] naturally results in a free energy $f(\phi, h)$ that depends on both $\phi$ and $h$, which is also covered in Appendix B.

This section ends the presentation of the special cases of the general model introduced. The following Conclusion includes a discussion of possible further extensions and open questions. Note that there are two Appendixes: Appendix A clarifies an issue in the comparison of the hydrodynamic long-wave approach and the present variational approach and Appendix B gives the variations of the energy functional in the most general case covered by the present work.

\section{CONCLUSION}

We have shown that a thin-film (or long-wave) model for the dynamics of liquid films on solid substrates with a free liquid-gas interface that is covered by soluble surfactants can be brought into a gradient dynamics form. Note that we always consider regimes where inertia does not enter (small Reynolds number). The gradient dynamics form is fully consistent with linear nonequilibrium thermodynamics including Onsager's reciprocity relations [3]. In the dilute limit, the model reduces

\footnotetext{
${ }^{9}$ It is our impression that the cross-coupling terms are often missing in the literature. This is also important on general grounds since without them the gradient dynamics structure of the dynamic equations is broken. We believe that this is the reason why Ref. [80] reports traveling and standing dewetting waves that are clearly unphysical in a relaxational setting. It seems also likely that the cusps in the dispersion curves obtained in Ref. [79] result from transitions between real and complex eigenvalues. The latter could again result from a broken gradient dynamics structure. However, the character of the eigenmodes is not explicitly mentioned in Ref. [79]; here we only deduce this possibility from the appearance of the dispersion curves.
} 
to the well known hydrodynamic form that includes Marangoni fluxes due to a linear equation of state relating surface tension and surfactant concentration at the free surface [5]. In this case the free-energy functional incorporates wetting energy (resulting in a Derjaguin or disjoining pressure), surface energy of the free interface [constant contribution plus entropic term, resulting in capillarity (Laplace pressure) and Marangoni flux], and bulk mixing free energy consisting solely of an (ideal-gas) entropic term that results in a dependence of evaporation on osmotic pressure but does not influence the convective flux. The entropic contributions also determine surfactant diffusion within and on the film and adsorption-desorption fluxes.

The advantage of the gradient dynamics form is that by amending the energy functional (incorporating nonentropic mixing and surface energies, bulk and surface rigidities, concentrationdependent wetting energies, etc.) one automatically obtains a thermodynamically consistent set of updated expressions for the Laplace and Derjaguin pressures; Marangoni, Korteweg, and diffusion fluxes; and evaporation as well as adsorption or desorption terms. There are also additional cross-coupling terms, e.g., in the case of a concentration-dependent wettability. The general model we have presented contains as limits the case of films of mixtures with components that are not surface active [27,28] and insoluble surfactants [29]. Such models with specific energies are furthermore found in Refs. [70,92] and [90,91], respectively. However, our work has also shown that many models existing in the literature are incomplete because they directly modify the hydrodynamic long-wave equations by incorporating, e.g., concentration-dependent Derjaguin pressures or nonlinear equations of state (for examples see Sec. IV and the discussions in Refs. [27-29]). Such ad hoc changes should be avoided as they alter only one transport channel (e.g., Marangoni flux or pressure gradient), while the underlying change of the energy functional affects all transport channels. So does, e.g., a change in the concentration dependence of the surface free energy which not only changes the surface equation of state and the Marangoni flux, but also affects surfactant diffusion and adsorption or desorption. A concentration-dependent wettability results in a concentration-dependent Derjaguin pressure and furthermore it gives an additional Marangoni-type flux, affects diffusion, evaporation, and adsorption or desorption. We expect that our general model with appropriately adapted energies can describe the film dynamics and incorporate the effects of, e.g., the spreading of patches of high-concentration surfactants on a liquid layer, which exhibit a local concentration maximum at the advancing surfactant front $[93,94]$, or the adsorption-desorption dynamics of nanoparticles that act as surfactant $[95,96]$.

Besides the amendments to the energy functional that we have discussed at length, an important element of a thermodynamically consistent gradient dynamics structure is the mobilities that form a positive-definite (positive entropy production) and symmetric (Onsager's reciprocity relations) matrix. Whenever a similar model for a relaxational situation is derived by making a long-wave approximation, a transformation into the gradient dynamics form should result in such a mobility matrix, thereby providing a valuable check that not all models in the literature pass. Here we have not changed the convective mobilities, but allowed for general diffusive ones $M(\phi)$ and $M_{\mathrm{s}}(\Gamma)$. A further discussion of the former $[M(\phi)]$ is found in Ref. [36], where a solvent-solute symmetric model is developed (without surface activity) that is also valid for high solute concentrations. However, the convective mobilities may also be amended: For instance, one can incorporate slip at the substrate or solvent diffusion along the substrate as discussed in Refs. [45,97] for films of simple liquids and layers of organic molecules, respectively. Less is known about the mobility coefficients of the nonconserved fluxes, so they are often approximated as constants. The influence of the mobilities should be further studied; in the present three-field case we expect a larger influence than in the one-field case of a film of simple liquid. There it was shown for the Plateau-Rayleigh instability of a ridge that the various (diffusive and convective) mobilities mainly change the relative timing of the different stages of the time evolution without much change to the pathway itself [97]. Models that allow for a crossover between diffusive and convective transport are discussed for monolayer solidification from liquid drops [98] and for the spreading of liquid drops on truly dry substrates where first an ultrathin adsorption layer (sometimes called precursor film) advances by diffusion before the mesoscopic drop spread by convection [99]. Another important factor that we have not 
discussed here is the dependence of the liquid viscosity on solute concentration. This is easy to incorporate, as long as the liquid is Newtonian. A further future task is the incorporation of surface viscosity [100] that should result in changes to the mobility matrix.

The gradient dynamics approach that we have presented may also be applied to situations where more than the three fields considered here (effective bulk solute height, projected surface concentration, and film height) matter. For example, systems with surfactant adsorption at the solid substrate have relevance, e.g., for chemically driven running droplets [101,102] where the transfer of a surfactant between different media and a solid substrate plays an important role. To model such systems one needs to account for adsorption at the substrate and diffusion of the adsorbate along the substrate. This can be achieved through the incorporation of a fourth field (adsorbate concentration) into the gradient dynamics structure and an appropriate amendment of the energy functional. This leads to a fourth evolution equation that couples through additional adsorption-desorption fluxes with the dynamics of the other fields. Such considerations are also important if one is seeking to model the dependence of the fluid dynamics in the contact line region on the concentration, including the concentration dependence of all the involved interfacial tensions and of the equilibrium contact angle. Such a model would allow one to describe the dynamics of effects such as surfactant-induced autophobing [103].

Another important extension is the incorporation of micelle dynamics [104,105]. This plays an important role, e.g., for superspreading, as does adsorption at the substrate [106-108]. To do this, one must again incorporate additional fields into the gradient dynamics approach. One could employ the free-energy approach of Ref. [109] and combine it with the present ideas to obtain coupled equations for the film height, effective solute height, effective micellar height, and surface concentrations. This is straightforward if the micelles are monodisperse in size. However, the number of equations will proliferate if the number of molecules per micelle is considered in detail. In hydrodynamic long-wave models only one size is normally considered [5,105,110].

Since the adsorption at the substrate may be physisorption or chemisorption, the question arises whether, in general, chemical reactions may be incorporated into a gradient dynamics. Reference [67] provides such a formulation for reaction-diffusion systems that may be coupled to the present formulation of thin-film hydrodynamics. Preliminary considerations show that this is possible and results, e.g., in cross couplings between chemical reactions and wettability. However, as briefly discussed in Sec. IIIE2, the correct way to construct the mobilities such that they agree with the ones obtained via kinetic considerations is still an open question.

Throughout the present work we have nearly exclusively referred to relaxational situations, i.e., experimental settings without any imposed influxes or throughflows of energy or mass, where the initial state relaxes towards a minimum of the underlying energy functional. However, the resulting gradient dynamics formulation for the time evolution can now be supplemented by well defined (normally nonvariational) terms to describe systems that are permanently out of equilibrium. Example of this are film flows and drop dynamics on inclined planes where a gradient dynamics model is obtained by incorporating the potential energy of the liquid into the energy functional [10]. Other examples include models for dip coating and Langmuir-Blodgett transfer processes where a film of solution or suspension is transferred from a bath onto a moving plate [31]. Then the relaxational gradient dynamics is supplemented by a dragging or comoving frame term that together with lateral boundary conditions representing the bath and the deposited layer, respectively, effectively transforms the model into a nonrelaxational out-of-equilibrium model that often shows multistability or self-organized pattern formation [31,91,111,112]. It is similar for dragged films of simple liquids (also known as the Landau-Levich problem) [113,114], films and drops on or in rotating cylinders [115,116], and evaporative dewetting of suspensions (in the comoving frame of a planar evaporation front) $[15,117]$.

Furthermore, one may impose certain influxes and/or outfluxes of material [118,119] or energy [30] that break the gradient dynamics structure. It should be further studied how the approach can be extended to systematically incorporate such imposed fluxes as well as internal sources, for example, the latent heat of evaporation. This also applies to applications to the 
modeling of interface-dominated out-of-equilibrium processes in (bio)active soft matter systems. For instance, Ref. [120] presents a model for the osmotic spreading dynamics of bacterial biofilms where a relaxational model for a mixture of aqueous solvent and biomass is supplemented by growth terms that model the proliferation of biomass. Another example considers a dilute carpet of insoluble self-propelled microswimmers on a liquid film and describes it using an extension of models developed for insoluble non-self-propelling surfactant particles [121,122]. To describe higher concentrations of the microswimmers one could employ the present model of soluble surfactants and add contributions resulting from the self-propulsion.

\section{ACKNOWLEDGMENTS}

We acknowledge discussions with many colleagues about the concept of gradient dynamics in the context of long-wave hydrodynamic models, for instance, Richard Craster, Oliver Jensen, Michael Shearer, and Tiezheng Qian. We thank the Center of Nonlinear Science and the Internationalisation Funds of the Westfälische Wilhelms Universität Münster for their support of our collaborative meetings and an extensive stay of LMP at Münster, respectively. Further we would like to thank the Isaac Newton Institute for Mathematical Sciences at the University of Cambridge for the Research Program "Mathematical Modelling and Analysis of Complex Fluids and Active Media in Evolving Domains", during which many discussions with colleagues took place and the first part of this work was conceived. We are thankful to Sarah Trinschek and Walter Tewes for triple-checking part of our calculations. U.T. also acknowledges discussions with Ofer Manor in the final stage of the project and GIF Grant No. I-1361-401.10/2016 for partial funding.

\section{APPENDIX A: ASYMPTOTIC LONG-WAVE EXPANSION VS VARIATIONAL APPROACH}

There is an interesting issue in the variational form of the evolution equations for an insoluble layer of surfactant on a liquid layer as presented in Ref. [29]. There, in Eq. (15) the Laplace pressure takes the form $-\partial_{x}\left(\gamma \partial_{x} h\right)$, where $\gamma=\gamma(\Gamma)$ is the surfactant concentration-dependent surface tension that emerges as the local grand potential.

Consider the curve representing the surface of a fluid in two dimensions with surface tension $\gamma=\gamma(s)$ as a function of arc length $s$. On mechanical grounds one should expect that the force on a curve element to be the derivative with respect to the arc length of $\gamma(s)$ t, i.e.,

$$
\frac{d}{d s}(\gamma(s) \mathbf{t})=\frac{d \gamma(s)}{d s} \mathbf{t}+\gamma(s) \frac{d \mathbf{t}}{d s}=\frac{d \gamma(s)}{d s} \mathbf{t}+\gamma(s) K \mathbf{n},
$$

where

$$
\mathbf{n}=\frac{1}{\xi}\left(-\partial_{x} h, 1\right)^{T}, \quad \mathbf{t}=\frac{1}{\xi}\left(1, \partial_{x} h\right)^{T}, \quad K=\frac{\partial_{x x} h}{\xi^{3}}
$$

are the normal vector, tangent vector, and curvature of the surface, respectively, and $\xi=[1+$ $\left.\left(\partial_{x} h\right)^{2}\right]^{1 / 2}$. This seems to indicate that the Laplace pressure term in a long-wave model should be $-\gamma \partial_{x x} h$ since Eq. (A1) gives the right-hand side of the classical hydrodynamic force boundary condition (BC) at a free surface, while the left-hand side is $\left(\boldsymbol{\tau}_{\text {in }}-\boldsymbol{\tau}_{\text {out }}\right) \cdot \mathbf{n}$.

We show next that the form $-\partial_{x}\left(\gamma \partial_{x} h\right)$ in Ref. [29] that also appears in all the models presented here naturally arises when projecting the force BC not onto $\mathbf{n}$ and $\mathbf{t}$ (as done for general interfaces), but onto the Cartesian unit vectors $\mathbf{e}_{x}=(1,0)^{T}$ and $\mathbf{e}_{z}=(0,1)^{T}$, as appropriate when performing a long-wave approximation. The stress tensor is

$$
\boldsymbol{\tau}=-p \mathbf{I}+\eta\left[\nabla \mathbf{v}+(\nabla \mathbf{v})^{T}\right]
$$


where $p(x, z)$ stands for the pressure field and $\mathbf{I}$ is the identity tensor. The force equilibrium is

$$
\left(\boldsymbol{\tau}-\boldsymbol{\tau}_{\text {air }}\right) \cdot \mathbf{n}=\gamma K \mathbf{n}+\left(\partial_{\mathrm{s}} \gamma\right) \mathbf{t},
$$

where the surface derivative is defined by $\partial_{\mathrm{s}}=\mathbf{t} \cdot \nabla$ and we assume that the ambient air does not transmit any shear stress $\left(\boldsymbol{\tau}_{\text {air }}=p_{\text {gas }} \mathbf{I}\right)$ and introduce $p=p_{\text {liq }}-p_{\text {gas }}$.

The boundary condition (A3) is of vectorial character, i.e., one can derive two scalar conditions by projecting it onto two different directions. In Refs. [4,5,123] projections onto $\mathbf{t}$ and $\mathbf{n}$ are used, resulting in, respectively,

$$
\begin{gathered}
\eta\left[\left(u_{z}+w_{x}\right)\left(1-h_{x}^{2}\right)+2\left(w_{z}-u_{x}\right) h_{x}\right]=\partial_{\mathrm{s}} \gamma\left(1+h_{x}^{2}\right), \\
p+\frac{2 \eta}{1+h_{x}^{2}}\left[-u_{x} h_{x}^{2}-w_{z}+h_{x}\left(u_{z}+w_{x}\right)\right]=-\gamma K .
\end{gathered}
$$

Note that to highest order in long-wave scaling (see below) this results in the BCs (when keeping all the surface tension terms) $p=-\varepsilon^{2} \gamma h_{x x}$ and $\eta u_{z}=\varepsilon \partial_{x} \gamma$.

Here, instead, we project onto $\mathbf{e}_{x}$ and $\mathbf{e}_{z}$ obtaining, respectively,

$$
\begin{gathered}
-h_{x}\left(2 \eta u_{x}-p\right)+\eta\left(u_{z}+w_{x}\right)=-h_{x} \gamma K+\partial_{\mathrm{s}} \gamma, \\
-\eta h_{x}\left(w_{x}+u_{z}\right)+2 \eta w_{z}-p=\gamma K+h_{x} \partial_{\mathrm{s}} \gamma .
\end{gathered}
$$

Next we introduce the long-wave scaling with length scale ratio $\varepsilon=H / L$. Note that we do not nondimensionalize. We also replace $K \approx h_{x x}$ and $\partial_{\mathrm{s}} \gamma \approx \partial_{x} \gamma$, formally introducing scaled (longwave) variables $x^{\prime}=\varepsilon x$ and $w^{\prime}=w / \varepsilon$. After dropping the primes we have, for $\mathbf{e}_{x}$ and $\mathbf{e}_{z}$ projections, respectively,

$$
\begin{gathered}
-\varepsilon h_{x}\left(2 \eta \varepsilon u_{x}-p\right)+\eta\left(u_{z}+\varepsilon^{2} w_{x}\right)=-\varepsilon^{3} \gamma h_{x} h_{x x}+\varepsilon \partial_{x} \gamma, \\
-\varepsilon \eta h_{x}\left(\varepsilon^{2} w_{x}+u_{z}\right)+2 \varepsilon \eta w_{z}-p=\varepsilon^{2} \gamma h_{x x}+\varepsilon^{2} h_{x} \partial_{x} \gamma .
\end{gathered}
$$

In the usual way [123] one takes into account that all velocities are small, introducing $u^{\prime}=u / \varepsilon$ and $w^{\prime}=w / \varepsilon$ and dropping small terms with the exception of surface tension related terms. After dropping the primes one has,

$$
\begin{aligned}
\varepsilon h_{x} p+\varepsilon \eta u_{z} & =-\varepsilon^{3} \gamma h_{x} h_{x x}+\varepsilon \partial_{x} \gamma, \\
-p & =\varepsilon^{2} \gamma h_{x x}+\varepsilon^{2} h_{x} \partial_{x} \gamma .
\end{aligned}
$$

Introducing Eq. (A11) into Eq. (A10), one has

$$
\varepsilon h_{x}\left(-\varepsilon^{2} \gamma h_{x x}-\varepsilon^{2} h_{x} \partial_{x} \gamma\right)+\varepsilon \eta u_{z}=-\varepsilon^{3} \gamma h_{x} h_{x x}+\varepsilon \partial_{x} \gamma,
$$

i.e.,

$$
\eta u_{z}=\left(1+\varepsilon^{2} h_{x}^{2}\right) \partial_{x} \gamma \approx \partial_{x} \gamma
$$

The second condition (A11) is identical to

$$
p=-\varepsilon^{2} \partial_{x}\left(\gamma \partial_{x} h\right)
$$

As the previous two equations give the $\mathrm{BC}$ for the bulk equations $u_{z z}=p_{x}$ and $p_{z}=0$, the involved quantities have to scale as $O\left(\varepsilon^{2} \gamma\right)=O\left(\partial_{x} \gamma\right)=O(p)=O(u)=O(1)$, in other words, $\partial_{x}\left(\gamma \partial_{x} h\right) \approx$ $\gamma \partial_{x x} h$. The difference is of higher order in $\varepsilon$. Our consideration poses the interesting question whether an asymptotic expansion should in general be done in such a way that it does not break deeper principles. Here the deeper principle is the thermodynamically consistent gradient dynamics formulation required for the description of a relaxational process. Therefore, $\partial_{x}\left(\gamma \partial_{x} h\right)$ should be preferred over $\gamma \partial_{x x} h$. 


\section{APPENDIX B: VARIATIONS IN THE GENERAL CASE}

The free energy $F[h, \Gamma, \phi]$ for the thin liquid film covered with soluble surfactant (i.e., a film of a mixture with surface active components) is

$$
F\left[h, \frac{\widetilde{\Gamma}}{\xi}, \frac{\psi}{h}\right]=\int\left\{h f\left(h, \frac{\psi}{h}\right)+g\left(h, \frac{\widetilde{\Gamma}}{\xi}, \frac{\psi}{h}\right)+\xi f_{\mathrm{s}}\left(h, \frac{\widetilde{\Gamma}}{\xi}\right)+h \frac{\kappa}{2}\left(\nabla \frac{\psi}{h}\right)^{2}+\frac{\kappa_{\mathrm{s}}}{2} \frac{1}{\xi}\left(\nabla \frac{\widetilde{\Gamma}}{\xi}\right)^{2}\right\} d A
$$

We define

$$
F\left[h, \frac{\widetilde{\Gamma}}{\xi}, \frac{\psi}{h}\right]=F_{\text {bulk }}+F_{\text {wet }}+F_{\text {surf }}+F_{\text {gradbulk }}+F_{\text {gradsurf }}
$$

and separately calculate the variations of the five terms in the free energy. For simplicity, we only consider the one-dimensional case. An extension to the general two-dimensional case is straightforward. Initially, we keep the full expression $\xi=\sqrt{1+\left(\partial_{x} h\right)^{2}}$ and introduce the long-wave approximation for $\xi$ later on. This implies

$$
\frac{\partial}{\partial h} \xi=0, \quad \frac{\partial \xi}{\partial\left(\partial_{x} h\right)}=\frac{1}{\xi} \partial_{x} h, \quad \partial_{x} \xi=\frac{1}{\xi}\left(\partial_{x} h\right)\left(\partial_{x x} h\right), \quad \frac{\partial}{\partial\left(\partial_{x} h\right)} \frac{1}{\xi}=-\frac{1}{\xi^{3}} \partial_{x} h
$$

\section{Variations with respect to $h$}

The variations with respect to $h$ are

$$
\begin{gathered}
\frac{\delta F_{\text {bulk }}}{\delta h}=f+h \partial_{h} f-\phi \partial_{\phi} f \\
\frac{\delta F_{\text {wet }}}{\delta h}=\partial_{h} g-\frac{\phi}{h} \partial_{\phi} g+\frac{d}{d x}\left[\frac{\Gamma}{\xi^{2}}\left(\partial_{\Gamma} g\right) \partial_{x} h\right] .
\end{gathered}
$$

Note that the final term was missed in Eq. (A4) of Ref. [29]. This then also results in amendments in their Eq. (23), namely, there is an additional $-\Gamma \partial_{\Gamma} g$ in the surface tension $\gamma$ in their Eq. (23) and the Marangoni force is $\nabla \gamma-\left(\partial_{\Gamma} g\right) \nabla \Gamma$ (note that our $g$ is their $f$ ).

Next we have

$$
\begin{aligned}
\frac{\delta F_{\text {surf }}}{\delta h} & =\xi \partial_{h} f_{\mathrm{s}}-\frac{d}{d x}\left[\frac{1}{\xi} f_{\mathrm{s}} \partial_{x} h-\frac{1}{\xi^{2}}\left(\partial_{\Gamma} f_{\mathrm{s}}\right) \widetilde{\Gamma} \partial_{x} h\right] \\
& =\xi \partial_{h} f_{\mathrm{s}}-\frac{d}{d x}\left[\frac{1}{\xi}\left(f_{\mathrm{s}}-\Gamma \partial_{\Gamma} f_{\mathrm{s}}\right) \partial_{x} h\right]
\end{aligned}
$$

For the next variation we need to use

$$
\frac{\delta\left(\int \star d x\right)}{\delta h}=\frac{\partial \star}{\partial h}-\frac{d}{d x} \frac{\partial \star}{\partial\left(\partial_{x} h\right)}+\frac{d^{2}}{d x^{2}} \frac{\partial \star}{\partial\left(\partial_{x x} h\right)} .
$$

We also need

$$
\begin{aligned}
\partial_{x} \frac{\widetilde{\Gamma}}{\xi} & =\frac{\partial_{x} \widetilde{\Gamma}}{\xi}-\frac{\widetilde{\Gamma}}{\xi^{2}} \partial_{x} \xi \\
& =\frac{\partial_{x} \widetilde{\Gamma}}{\xi}-\frac{\widetilde{\Gamma}}{\xi^{3}}\left(\partial_{x} h\right)\left(\partial_{x x} h\right) .
\end{aligned}
$$


The variations of the gradient terms are then

$$
\begin{aligned}
\frac{\delta F_{\text {gradbulk }}}{\delta h} & =\frac{\kappa}{2}\left(\partial_{x} \frac{\psi}{h}\right)^{2}+\kappa\left(\partial_{x} \frac{\psi}{h}\right)\left[-\frac{\partial_{x} \psi}{h}+\frac{2 \psi}{h^{2}} \partial_{x} h\right]+\frac{d}{d x}\left[\kappa \frac{\psi}{h}\left(\partial_{x} \frac{\psi}{h}\right)\right] \\
& =\frac{\kappa}{2}\left(\partial_{x} \phi\right)^{2}+\kappa \frac{\phi}{h}\left(\partial_{x} h\right)\left(\partial_{x} \phi\right)+\kappa \phi \partial_{x x} \phi
\end{aligned}
$$

and

$$
\begin{aligned}
\frac{\delta F_{\text {gradsurf }}}{\delta h}= & -\frac{d}{d x}\left[-\frac{\kappa_{\mathrm{s}}}{2}\left(\partial_{x} \frac{\widetilde{\Gamma}}{\xi}\right)^{2} \frac{\partial_{x} h}{\xi^{3}}-\frac{\kappa_{\mathrm{s}}}{\xi^{4}}\left(\partial_{x} \widetilde{\Gamma} \partial_{x} h+\widetilde{\Gamma} \partial_{x x} h-3 \frac{\widetilde{\Gamma}}{\xi^{2}}\left(\partial_{x} h\right)^{2} \partial_{x x} h\right) \partial_{x} \frac{\widetilde{\Gamma}}{\xi}\right] \\
& -\frac{d^{2}}{d x^{2}}\left[\frac{\kappa_{\mathrm{s}}}{\xi^{4}}\left(\partial_{x} \frac{\widetilde{\Gamma}}{\xi}\right) \widetilde{\Gamma} \partial_{x} h\right] \\
= & -\frac{d}{d x}\left\{\frac { \kappa _ { \mathrm { s } } } { \xi ^ { 3 } } \left[-\frac{1}{2}\left(\partial_{x} \Gamma\right)^{2} \partial_{x} h-\left(\partial_{x} \Gamma \partial_{x} h+\Gamma \partial_{x x} h-2 \frac{\Gamma}{\xi^{2}}\left(\partial_{x} h\right)^{2} \partial_{x x} h\right) \partial_{x} \Gamma\right.\right. \\
& \left.\left.-\left(3 \frac{\Gamma}{\xi^{2}}\left(\partial_{x} h\right)^{2} \partial_{x x} h-\partial_{x} \Gamma \partial_{x} h-\Gamma \partial_{x x} h\right) \partial_{x} \Gamma+\Gamma \partial_{x} h \partial_{x x} \Gamma\right]\right\} \\
= & \frac{d}{d x}\left\{\frac{\kappa_{\mathrm{s}}}{\xi^{3}}\left[\frac{1}{2}\left(\partial_{x} \Gamma\right)^{2} \partial_{x} h+\frac{\Gamma}{\xi^{2}}\left(\partial_{x} h\right)^{2}\left(\partial_{x x} h\right) \partial_{x} \Gamma-\Gamma \partial_{x} h \partial_{x x} \Gamma\right]\right\}
\end{aligned}
$$

\section{Variations with respect to $\tilde{\Gamma}$}

The variations with respect to $\widetilde{\Gamma}$ are

$$
\begin{gathered}
\frac{\delta F_{\text {bulk }}}{\delta \widetilde{\Gamma}}=0, \quad \frac{\delta F_{\text {gradbulk }}}{\delta \widetilde{\Gamma}}=0, \\
\frac{\delta F_{\text {wet }}}{\delta \widetilde{\Gamma}}=\frac{1}{\xi} \partial_{\Gamma} g, \\
\frac{\delta F_{\text {surf }}}{\delta \widetilde{\Gamma}}=\partial_{\Gamma} f_{\mathrm{s}}, \\
\frac{\delta F_{\text {gradsurf }}}{\delta \widetilde{\Gamma}}=-\kappa_{\mathrm{s}} \frac{1}{\xi^{4}}\left(\partial_{x} \Gamma\right)\left(\partial_{x} h\right)\left(\partial_{x x} h\right)-\kappa_{\mathrm{s}} \frac{d}{d x}\left[\frac{1}{\xi^{2}} \partial_{x} \Gamma\right] \\
=\kappa_{\mathrm{s}} \frac{1}{\xi^{4}}\left(\partial_{x} \Gamma\right)\left(\partial_{x} h\right)\left(\partial_{x x} h\right)-\kappa_{\mathrm{s}} \frac{1}{\xi^{2}} \partial_{x x} \Gamma .
\end{gathered}
$$

\section{Variations with respect to $\psi$}

The variations with respect to $\psi$ are

$$
\begin{gathered}
\frac{\delta F_{\text {surf }}}{\delta \psi}=0, \quad \frac{\delta F_{\text {gradsurf }}}{\delta \psi}=0, \\
\frac{\delta F_{\text {wet }}}{\delta \psi}=\frac{1}{h} \partial_{\phi} g, \\
\frac{\delta F_{\text {bulk }}}{\delta \psi}=\partial_{\phi} f, \\
\frac{\delta F_{\text {gradbulk }}}{\delta \psi}=-\kappa \frac{1}{h}\left(\partial_{x} \phi\right)\left(\partial_{x} h\right)-\kappa \partial_{x x} \phi .
\end{gathered}
$$




\section{Collecting the terms}

The resulting expressions for the variations are

$$
\begin{gathered}
p=\frac{\delta F}{\delta h}=f+h \partial_{h} f-\phi \partial_{\phi} f+\partial_{h} g-\frac{\phi}{h} \partial_{\phi} g+\xi \partial_{h} f_{\mathrm{s}} \\
+\frac{\kappa}{2}\left(\partial_{x} \phi\right)^{2}+\kappa \frac{\phi}{h}\left(\partial_{x} h\right)\left(\partial_{x} \phi\right)+\kappa \phi \partial_{x x} \phi \\
-\partial_{x}\left\{\frac{1}{\xi}\left[f_{\mathrm{s}}-\Gamma \partial_{\Gamma} f_{\mathrm{s}}-\frac{\Gamma}{\xi} \partial_{\Gamma} g-\frac{\kappa_{\mathrm{s}}}{2 \xi^{2}}\left(\partial_{x} \Gamma\right)^{2}+\frac{\kappa_{\mathrm{s}}}{\xi} \Gamma \partial_{x}\left(\frac{1}{\xi} \partial_{x} \Gamma\right)\right] \partial_{x} h\right\} \\
\mu_{\mathrm{s}}=\frac{\delta F}{\delta \widetilde{\Gamma}}=\frac{1}{\xi} \partial_{\Gamma} g+\partial_{\Gamma} f_{\mathrm{s}}-\frac{\kappa_{\mathrm{s}}}{\xi} \partial_{x}\left(\frac{1}{\xi} \partial_{x} \Gamma\right), \\
\mu=\frac{\delta F}{\delta \psi}=\frac{1}{h} \partial_{\phi} g+\partial_{\phi} f-\frac{\kappa}{h} \partial_{x}\left(h \partial_{x} \phi\right) .
\end{gathered}
$$

This seems the appropriate stage in the derivation to apply the long-wave approximation, i.e., to use $\left(\partial_{x} h\right)^{2} \sim \varepsilon^{2} \ll 1$. Therefore $\xi \approx 1+O\left(\varepsilon^{2}\right)$ and one obtains to highest order

$$
\begin{gathered}
p=\frac{\delta F}{\delta h}=f+h \partial_{h} f-\phi \partial_{\phi} f+\partial_{h} g-\frac{\phi}{h} \partial_{\phi} g+\partial_{h} f_{\mathrm{s}} \\
+\frac{\kappa}{2}\left(\partial_{x} \phi\right)^{2}+\kappa \frac{\phi}{h}\left(\partial_{x} h\right)\left(\partial_{x} \phi\right)+\kappa \phi \partial_{x x} \phi-\partial_{x}\left[\tilde{\omega} \partial_{x} h,\right] \\
\mu_{\mathrm{s}}=\frac{\delta F}{\delta \widetilde{\Gamma}}=\partial_{\Gamma}\left(f_{\mathrm{s}}+g\right)-\kappa_{\mathrm{s}} \partial_{x x} \Gamma \\
\mu=\frac{\delta F}{\delta \psi}=\partial_{\phi} f+\frac{1}{h} \partial_{\phi} g-\frac{\kappa}{h} \partial_{x}\left(h \partial_{x} \phi\right)
\end{gathered}
$$

where we have introduced

$$
\tilde{\gamma}=\tilde{\omega}=f_{\mathrm{s}}-\Gamma \partial_{\Gamma} f_{\mathrm{s}}-\Gamma \partial_{\Gamma} g-\frac{\kappa_{\mathrm{s}}}{2}\left(\partial_{x} \Gamma\right)^{2}+\kappa_{\mathrm{s}} \Gamma \partial_{x x} \Gamma,
$$

corresponding to the surface grand potential density for the nonlocal case. Note that $\nabla \tilde{\gamma}=-\Gamma \nabla \mu_{s}-$ $\partial_{\Gamma} \nabla \Gamma$. The free energy in the general case (B1) may be simplified by assuming that cross couplings between composition and film height are all contained in $g(h, \Gamma, \phi)$ and do not appear in the bulk and surface energy. The latter are then $f(\phi)$ and $f_{\mathrm{s}}(\Gamma)$, respectively. In consequence, $\partial_{h} f=0$ and $\partial_{h} f_{\mathrm{s}}=0$ Eqs. (B24)-(B26) simplify accordingly. The general expressions for the variations, i.e., Eqs. (B24)-(B26), are then introduced into the general gradient dynamics form (42)-(45). With specific simplifying assumptions for the individual terms of the energy functional, one obtains several models in the literature and all the models introduced above as special cases.

[1] L. Onsager, Reciprocal relations in irreversible processes. I, Phys. Rev. 37, 405 (1931).

[2] L. Onsager, Reciprocal relations in irreversible processes. II, Phys. Rev. 38, 2265 (1931).

[3] M. Doi, Onsager's variational principle in soft matter, J. Phys.: Condens. Matter 23, 284118 (2011).

[4] A. Oron, S. H. Davis, and S. G. Bankoff, Long-scale evolution of thin liquid films, Rev. Mod. Phys. 69, 931 (1997).

[5] R. V. Craster and O. K. Matar, Dynamics and stability of thin liquid films, Rev. Mod. Phys. 81, 1131 (2009).

[6] V. S. Mitlin, Dewetting of solid surface: Analogy with spinodal decomposition, J. Colloid Interface Sci. 156, 491 (1993). 


\section{GRADIENT DYNAMICS MODELS FOR LIQUID FILMS ...}

[7] A. Oron and P. Rosenau, Formation of patterns induced by thermocapillarity and gravity, J. Phys. (France) II 2, 131 (1992).

[8] U. Thiele, Thin film evolution equations from (evaporating) dewetting liquid layers to epitaxial growth, J. Phys.: Condens. Matter 22, 084019 (2010).

[9] D. Bonn, J. Eggers, J. Indekeu, J. Meunier, and E. Rolley, Wetting and spreading, Rev. Mod. Phys. 81, 739 (2009).

[10] S. Engelnkemper, M. Wilczek, S. V. Gurevich, and U. Thiele, Morphological transitions of sliding drops-Dynamics and bifurcations, Phys. Rev. Fluids 1, 073901 (2016).

[11] J. W. Cahn and J. E. Hilliard, Free energy of a nonuniform system. 1. Interfacual free energy, J. Chem. Phys. 28, 258 (1958).

[12] J. W. Cahn, Phase separation by spinodal decomposition in isotropic systems, J. Chem. Phys. 42, 93 (1965).

[13] J. S. Langer, in Solids far from Equilibrium, edited by C. Godreche (Cambridge University Press, Cambridge, 1992), pp. 297-363.

[14] L. M. Pismen and Y. Pomeau, Disjoining potential and spreading of thin liquid layers in the diffuse interface model coupled to hydrodynamics, Phys. Rev. E 62, 2480 (2000).

[15] U. Thiele, Patterned deposition at moving contact line, Adv. Colloid Interface Sci. 206, 399 (2014).

[16] B. J. Spencer, P. W. Voorhees, and S. H. Davis, Morphological Instability in Epitaxially Strained Dislocation-Free Solid Films, Phys. Rev. Lett. 67, 3696 (1991).

[17] A. A. Golovin, S. H. Davis, and A. A. Nepomnyashchy, Model for faceting in a kinetically controlled crystal growth, Phys. Rev. E 59, 803 (1999).

[18] A. A. Golovin, M. S. Levine, T. V. Savina, and S. H. Davis, Faceting instability in the presence of wetting interactions: A mechanism for the formation of quantum dots, Phys. Rev. B 70, 235342 (2004).

[19] D. D. Vvedensky, Multiscale modeling of nanostructures, J. Phys.: Condens. Matter 16, R1537 (2004).

[20] D. Gallez, N. M. Costa Pinto, and P. M. Bisch, Nonlinear dynamics and rupture of lipid bilayers, J. Colloid Interface Sci. 160, 141 (1993).

[21] U. M. B. Marconi and P. Tarazona, Dynamic density functional theory of fluids, J. Chem. Phys. 110, 8032 (1999).

[22] U. M. B. Marconi and P. Tarazona, Dynamic density functional theory of fluids, J. Phys.: Condens. Matter 12, A413 (2000).

[23] A. J. Archer and R. Evans, Dynamical density functional theory and its application to spinodal decomposition, J. Chem. Phys. 121, 4246 (2004).

[24] A. J. Archer and M. Rauscher, Dynamical density functional theory for interacting Brownian particles: Stochastic or deterministic? J. Phys. A: Math. Gen. 37, 9325 (2004).

[25] A. Pototsky, M. Bestehorn, D. Merkt, and U. Thiele, Alternative pathways of dewetting for a thin liquid two-layer film, Phys. Rev. E 70, 025201(R) (2004).

[26] S. Jachalski, R. Huth, G. Kitavtsev, D. Peschka, and B. Wagner, Stationary solutions of liquid two-layer thin-film models, SIAM J. Appl. Math. 73, 1183 (2013).

[27] U. Thiele, Note on thin film equations for solutions and suspensions, Eur. Phys. J. Spec. Top. 197, 213 (2011).

[28] U. Thiele, D. V. Todorova, and H. Lopez, Gradient Dynamics Description for Films of Mixtures and Suspensions: Dewetting Triggered by Coupled Film Height and Concentration Fluctuations, Phys. Rev. Lett. 111, 117801 (2013).

[29] U. Thiele, A. J. Archer, and M. Plapp, Thermodynamically consistent description of the hydrodynamics of free surfaces covered by insoluble surfactants of high concentration, Phys. Fluids 24, 102107 (2012).

[30] A. Pototsky, M. Bestehorn, D. Merkt, and U. Thiele, Morphology changes in the evolution of liquid two-layer films, J. Chem. Phys. 122, 224711 (2005).

[31] M. Wilczek, W. B. H. Tewes, S. V. Gurevich, M. H. Köpf, L. Chi, and U. Thiele, Modelling pattern formation in dip-coating experiments, Math. Model. Nat. Phenom. 10, 44 (2015).

[32] C. Sample and A. A. Golovin, Nonlinear dynamics of a double bilipid membrane, Phys. Rev. E 76, 031925 (2007). 
[33] Y. Hirose, S. Komura, and D. Andelman, Concentration fluctuations and phase transitions in coupled modulated bilayers, Phys. Rev. E 86, 021916 (2012).

[34] A. J. Archer, Dynamical density functional theory: Binary phase-separating colloidal fluid in a cavity, J. Phys.: Condens. Matter 17, 1405 (2005).

[35] A. J. Archer, M. J. Robbins, and U. Thiele, Dynamical density functional theory for the dewetting of evaporating thin films of nanoparticle suspensions exhibiting pattern formation, Phys. Rev. E 81, 021602 (2010).

[36] X. Xu, U. Thiele, and T. Qian, A variational approach to thin film hydrodynamics of binary mixtures, J. Phys.: Condens. Matter 27, 085005 (2015).

[37] H. Emmerich, H. Löwen, R. Wittkowski, T. Gruhn, G. I. Toth, G. Tegze, and L. Granasy, Phase-fieldcrystal models for condensed matter dynamics on atomic length and diffusive time scales: An overview, Adv. Phys. 61, 665 (2012).

[38] Y. Hirose, S. Komura, and D. Andelman, Coupled modulated bilayers: A phenomenological model, Chem. Phys. Chem. 10, 2839 (2009).

[39] M. Doi, Soft Matter Physics (Oxford University Press, Oxford, 2013).

[40] P.-G. de Gennes, Wetting: Statics and dynamics, Rev. Mod. Phys. 57, 827 (1985).

[41] J. N. Israelachvili, Intermolecular and Surface Forces, 3rd ed. (Academic, London, 2011).

[42] Thin Films of Soft Matter, edited by S. Kalliadasis and U. Thiele (Springer, Wien, 2007).

[43] N. Tretyakov, M. Müller, D. Todorova, and U. Thiele, Parameter passing between molecular dynamics and continuum models for droplets on solid substrates: The static case, J. Chem. Phys. 138, 064905 (2013).

[44] A. P. Hughes, U. Thiele, and A. J. Archer, Liquid drops on a surface: using density functional theory to calculate the binding potential and drop profiles and comparing with results from mesoscopic modeling, J. Chem. Phys. 142, 074702 (2015).

[45] A. Münch, B. Wagner, and T. P. Witelski, Lubrication models with small to large slip lengths, J. Eng. Math. 53, 359 (2005).

[46] A. V. Lyushnin, A. A. Golovin, and L. M. Pismen, Fingering instability of thin evaporating liquid films, Phys. Rev. E 65, 021602 (2002).

[47] S. Bommer, F. Cartellier, S. Jachalski, D. Peschka, R. Seemann, and B. Wagner, Droplets on liquids and their journey into equilibrium, Eur. Phys. J. E 36, 87 (2013).

[48] N. Clarke, Toward a model for pattern formation in ultrathin-film binary mixtures, Macromolecules 38, 6775 (2005).

[49] K. R. Thomas, N. Clarke, R. Poetes, M. Morariu, and U. Steiner, Wetting induced instabilities in miscible polymer blends, Soft Matter 6, 3517 (2010).

[50] A. J. Archer, D. Pini, R. Evans, and L. Reatto, Model colloidal fluid with competing interactions: Bulk and interfacial properties, J. Chem. Phys. 126, 014104 (2007).

[51] A. J. Archer, C. Ionescu, D. Pini, and L. Reatto, Theory for the phase behavior of a colloidal fluid with competing interactions, J. Phys.: Condens. Matter 20, 415106 (2008).

[52] J.-P. Hansen and I. R. McDonald, Theory of Simple Liquids, 3rd ed. (Academic, London, 2006).

[53] Y. Pawar and K. J. Stebe, Marangoni effects on drop deformation in an extensional flow: The role of surfactant physical chemistry.1. insoluble surfactants, Phys. Fluids 8, 1738 (1996).

[54] Y. W. Kruijt-Stegeman, F. N. van de Vosse, and H. E. H. Meijer, Droplet behavior in the presence of insoluble surfactants, Phys. Fluids 16, 2785 (2004).

[55] C. D. Eggleton, Y. P. Pawar, and K. J. Stebe, Insoluble surfactants on a drop in an extensional flow: A generalization of the stagnated surface limit to deforming interfaces, J. Fluid Mech. 385, 79 (1999).

[56] D. Jasnow and J. Viñals, Coarse-grained description of thermo-capillary flow, Phys. Fluids 8, 660 (1996).

[57] U. Thiele, S. Madruga, and L. Frastia, Decomposition driven interface evolution for layers of binary mixtures: I. Model derivation and stratified base states, Phys. Fluids 19, 122106 (2007).

[58] G. L. Leal, Advanced Transport Phenomena (Cambridge University Press, Cambridge, 2007).

[59] P. Atkins and J. de Paula, Atkins' Physical Chemistry, 9th ed. (Oxford University Press, Oxford, 2010).

[60] A. Rednikov and P. Colinet, Singularity-free description of moving contact lines for volatile liquids, Phys. Rev. E 87, 010401 (2013). 
[61] V. S. Ajaev and G. M. Homsy, Steady vapor bubbles in rectangular microchannels, J. Colloid Interface Sci. 240, 259 (2001).

[62] V. S. Ajaev, Evolution of dry patches in evaporating liquid films, Phys. Rev. E 72, 031605 (2005).

[63] A. Y. Rednikov and P. Colinet, Vapor-liquid steady meniscus at a superheated wall: Asymptotics in an intermediate zone near the contact line, Microgravity Sci. Technol. 22, 249 (2010).

[64] H. Diamant and D. Andelman, Kinetics of surfactant adsorption at fluid-fluid interfaces, J. Phys. Chem. 100, 13732 (1996).

[65] V. B. Fainerman, S. A. Zholob, E. H. Lucassen-Reynders, and R. Miller, Comparison of various models describing the adsorption of surfactant molecules capable of interfacial reorientation, J. Colloid Interface Sci. 261, 180 (2003).

[66] J. K. Ferri and K. J. Stebe, Soluble surfactants undergoing surface phase transitions: A Maxwell construction and the dynamic surface tension, J. Colloid Interface Sci. 209, 1 (1999).

[67] A. Mielke, A gradient structure for reaction-diffusion systems and for energy-drift-diffusion systems, Nonlinearity 24, 1329 (2011).

[68] O. E. Jensen and J. B. Grotberg, The spreading of heat or soluble surfactant along a thin liquid film, Phys. Fluids A 5, 58 (1993).

[69] M. R. E. Warner, R. V. Craster, and O. K. Matar, Surface patterning via evaporation of ultrathin films containing nanoparticles, J. Colloid Interface Sci. 267, 92 (2003).

[70] L. Ó. Náraigh and J. L. Thiffeault, Nonlinear dynamics of phase separation in thin films, Nonlinearity 23, 1559 (2010).

[71] R. Borcia, S. Menzel, M. Bestehorn, S. Karpitschka, and H. Riegler, Delayed coalescence of droplets with miscible liquids: Lubrication and phase field theories, Eur. Phys. J. E 34, 24 (2011).

[72] S. Karpitschka and H. Riegler, Sharp transition between coalescence and non-coalescence of sessile drops, J. Fluid Mech. 743, R1 (2014).

[73] M. R. E. Warner, R. V. Craster, and O. K. Matar, Fingering phenomena created by a soluble surfactant deposition on a thin liquid film, Phys. Fluids 16, 2933 (2004).

[74] M. S. Borgas and J. B. Grotberg, Monolayer flow on a thin film (lung application), J. Fluid Mech. 193, 151 (1988).

[75] D. P. Gaver and J. B. Grotberg, The dynamics of a localized surfactant on a thin film, J. Fluid Mech. 213, 127 (1990).

[76] O. K. Matar and R. V. Craster, Models for Marangoni drying, Phys. Fluids 13, 1869 (2001).

[77] M. Hanyak, D. K. N. Sinz, and A. A. Darhuber, Soluble surfactant spreading on spatially confined thin liquid films, Soft Matter 8, 7660 (2012).

[78] M. R. E. Warner, R. V. Craster, and O. K. Matar, Dewetting of ultrathin surfactant-covered films, Phys. Fluids 14, 4040 (2002).

[79] G. H. Hu, Linear stability of ultrathin slipping films with insoluble surfactant, Phys. Fluids 17, 088105 (2005).

[80] L. S. Fisher and A. A. Golovin, Instability of a two-layer thin liquid film with surfactants: Dewetting waves, J. Colloid Interface Sci. 307, 203 (2007).

[81] C. X. Li, K. Jiang, and X. M. Ye, Stability characteristics of thin film dewetting with insoluble surfactant, Acta Phys. Sin. 62, 234702 (2013).

[82] G. H. Hu, Influences of oscillatory structural forces on dewetting of nanoparticle-laden ultra-thin films, Acta Mech. Sin. 28, 737 (2012).

[83] R. V. Craster and O. K. Matar, On autophobing in surfactant-driven thin films, Langmuir 23, 2588 (2007).

[84] R. Fondecave and F. Brochard-Wyart, Wetting laws for polymer solutions, Europhys. Lett. 37, 115 (1997).

[85] R. Fondecave and F. Brochard-Wyart, Polymers as dewetting agents, Macromolecules 31, 9305 (1998).

[86] B. V. Derjaguin and N. V. Churaev, Disjoining pressure of thin-layers of binary-solutions, J. Colloid Interface Sci. 62, 369 (1977).

[87] M. Geoghegan and G. Krausch, Wetting at polymer surfaces and interfaces, Prog. Polym. Sci. 28, 261 (2003).

[88] H. Riegler and K. Spratte, Structural-changes in lipid monolayers during the Langmuir-Blodgett transfer due to substrate monolayer interactions, Thin Solid Films 210, 9 (1992). 
[89] L. Q. Li, M. H. Köpf, S. V. Gurevich, R. Friedrich, and L. F. Chi, Structure formation by dynamic self-assembly, Small 8, 488 (2012).

[90] M. H. Köpf, S. V. Gurevich, and R. Friedrich, Thin film dynamics with surfactant phase transition, Europhys. Lett. 86, 66003 (2009).

[91] M. H. Köpf, S. V. Gurevich, R. Friedrich, and L. F. Chi, Pattern formation in monolayer transfer systems with substrate-mediated condensation, Langmuir 26, 10444 (2010).

[92] C. K. Sarika, G. Tomar, J. K. Basu, and U. Thiele, Bimodality of hierarchical self-assembly of polymeric nanoparticles, Soft Matter 11, 8975 (2015).

[93] D. W. Fallest, A. M. Lichtenberger, C. J. Fox, and K. E. Daniels, Fluorescent visualization of a spreading surfactant, New J. Phys. 12, 073029 (2010).

[94] S. L. Strickland, M. Hin, M. R. Sayanagi, C. Gaebler, K. E. Daniels, and R. Levy, Self-healing dynamics of surfactant coatings on thin viscous films, Phys. Fluids 26, 042109 (2014).

[95] B. P. Binks, Particles as surfactants-Similarities and differences, Curr. Opin. Colloid Interface Sci. 7, 21 (2002).

[96] V. Garbin, J. C. Crocker, and K. J. Stebe, Forced desorption of nanoparticles from an oil-water interface, Langmuir 28, 1663 (2012).

[97] C. Honisch, T.-S. Lin, A. Heuer, U. Thiele, and S. V. Gurevich, Instabilities of layers of deposited molecules on chemically stripe patterned substrates: Ridges vs. drops, Langmuir 31, 10618 (2015).

[98] A. Yochelis and L. M. Pismen, Droplet motion driven by surface freezing or melting: A mesoscopic hydrodynamic approach, Phys. Rev. E 72, 025301(R) (2005).

[99] H. Yin, D. N. Sibley, U. Thiele, and A. J. Archer, Films, layers and droplets: The effect of near-wall fluid structure on spreading dynamics, arXiv:1611.00390.

[100] B. Scheid, J. Delacotte, B. Dollet, E. Rio, F. Restagno, E. A. van Nierop, I. Cantat, D. Langevin, and H. A. Stone, The role of surface rheology in liquid film formation, Europhys. Lett. 90, 24002 (2010).

[101] Y. Sumino, M. Nagayama, H. Kitahata, S. M. Nomura, N. Magome, Y. Mori, and K. Yoshikawa, Chemo-sensitive running droplet, Phys. Rev. E 72, 041603 (2005).

[102] Y. Sumino, N. Magome, and K. Yoshikawa, An oil droplet that spontaneously climbs up stairs, Prog. Theor. Phys. Suppl. 161, 348 (2006).

[103] B. Bera, M. H. G. Duits, M. A. C. Stuart, D. van den Ende, and F. Mugele, Surfactant induced autophobing, Soft Matter 12, 4562 (2016).

[104] R. V. Craster and O. K. Matar, Numerical simulations of fingering instabilities in surfactant-driven thin films, Phys. Fluids 18, 032103 (2006).

[105] B. D. Edmonstone, R. V. Craster, and O. K. Matar, Surfactant-induced fingering phenomena beyond the critical micelle concentration, J. Fluid Mech. 564, 105 (2006).

[106] G. Karapetsas, R. V. Craster, and O. K. Matar, On surfactant-enhanced spreading and superspreading of liquid drops on solid surfaces, J. Fluid Mech. 670, 5 (2011).

[107] A. Nikolov and D. Wasan, Superspreading mechanisms: An overview, Eur. Phys. J. Spec. Top. 197, 325 (2011).

[108] C. Maldarelli, On the microhydrodynamics of superspreading, J. Fluid Mech. 670, 1 (2011).

[109] R. Hadgiivanova, H. Diamant, and D. Andelman, Kinetics of surfactant micellization: A free energy approach, J. Phys. Chem. B 115, 7268 (2011).

[110] D. R. Beacham, O. K. Matar, and R. V. Craster, Surfactant-enhanced rapid spreading of drops on solid surfaces, Langmuir 25, 14174 (2009).

[111] M. H. Köpf, S. V. Gurevich, R. Friedrich, and U. Thiele, Substrate-mediated pattern formation in monolayer transfer: A reduced model, New J. Phys. 14, 023016 (2012).

[112] M. H. Köpf and U. Thiele, Emergence of the bifurcation structure of a Langmuir-Blodgett transfer model, Nonlinearity 27, 2711 (2014).

[113] J. H. Snoeijer, J. Ziegler, B. Andreotti, M. Fermigier, and J. Eggers, Thick Films of Viscous Fluid Coating a Plate Withdrawn from a Liquid Reservoir, Phys. Rev. Lett. 100, 244502 (2008).

[114] M. Galvagno, D. Tseluiko, H. Lopez, and U. Thiele, Continuous and Discontinuous Dynamic Unbinding Transitions in Drawn Film Flow, Phys. Rev. Lett. 112, 137803 (2014).

[115] H. K. Moffatt, Behavior of a viscous film on outer surface of a rotating cylinder, J. Méc. 16, 651 (1977). 
[116] T.-S. Lin, S. Rogers, D. Tseluiko, and U. Thiele, Bifurcation analysis of the behavior of partially wetting liquids on a rotating cylinder, Phys. Fluids 28, 082102 (2016).

[117] L. Frastia, A. J. Archer, and U. Thiele, Modelling the formation of structured deposits at receding contact lines of evaporating solutions and suspensions, Soft Matter 8, 11363 (2012).

[118] M. Bestehorn and D. Merkt, Regular Surface Patterns on Rayleigh-Taylor Unstable Evaporating Films Heated from Below, Phys. Rev. Lett. 97, 127802 (2006).

[119] Y. Tsoumpas, S. Dehaeck, M. Galvagno, A. Rednikov, H. Ottevaere, U. Thiele, and P. Colinet, Nonequilibrium Gibbs' criterion for completely wetting volatile liquids, Langmuir 30, 11847 (2014).

[120] S. Trinschek, K. John, and U. Thiele, From a thin film model for passive suspensions towards the description of osmotic biofilm spreading, AIMS Mater. Sci. 3, 1138 (2016).

[121] S. Alonso and A. S. Mikhailov, Towards active microfluidics: Interface turbulence in thin liquid films with floating molecular machines, Phys. Rev. E 79, 061906 (2009).

[122] A. Pototsky, U. Thiele, and H. Stark, Mode instabilities and dynamic patterns in a colony of self-propelled surfactant particles covering a thin liquid layer, Eur. Phys. J. E 39, 1 (2016).

[123] U. Thiele, in Thin Films of Soft Matter, edited by S. Kalliadasis and U. Thiele (Springer, Wien, 2007), p. 25. 